\title{
expérimentation en vraie grandeur d'un rideau de palplanches
}

\author{
par \\ J.P. Gigan \\ Ingénieur civil des Ponts et Chaussées \\ Laboratoire régional de l'équipement - Le Bourget - France
}

\section{Introduction}

A côté des méthodes classiques aux états limites, les méthodes dites "élastoplastiques " sont de plus en plus utilisées pour le calcul des rideaux de palplanches.

L'étude du comportement réel de ces écrans de soutènement a pour objectif de nous renseigner à la fois sur la répartition des efforts dans l'Ouvrage et sur les lois de mobilisation des pressions du sol.

Dans ce but, plusieurs expérimentations ont été réalisées par les laboratoires des Ponts et Chaussées depuis 1974. (Josseaume et Al. 1977 - Gigan 1979). Celle qui est présentée ci-après concerne un rideau ancré par des tirants précontraints inclinés et a permis d'étudier en particulier l'intéraction rideau-ancrage.

Après une description du site et de l'équipement des palplanches, nous présenterons l'ensemble des mesures et l'interprétation qui en a été faite, avant de comparer ces résultats expérimentaux à ceux d'un calcul complet de l'ouvrage par une méthode "élastoplastique ».

\section{Présentation du site et de l'ouvrage}

\subsection{L'ouvrage}

La réalisation de la Voie rive gauche de Seine, en aval de Paris a nécessité la construction d'un rideau de palplanches le long des berges du fleuve, sur un longueur de 700 mètres environ entre les communes d'Asnières et de Villeneuve-la-Garenne.

La coupe transversale de l'Ouvrage (fig. 1) au niveau des palplanches expérimentales en fixe les principales caractéristiques :

- Palplanches LARSSEN VS de 17,2 mètres de langueur totale.

- Tirants d'ancrage IRP 10 T 13 d'une capacité de 93 tonnes, inclinés à $30^{\circ}$, espacés de deux mètres. Ces tirants ont une longueur de 24 mètres dont 10 mètres de scellement.

- Le rideau est surmonté d'un mur de couronnement en béton, portant la hauteur totale du soutènement à près de 10 mètres.

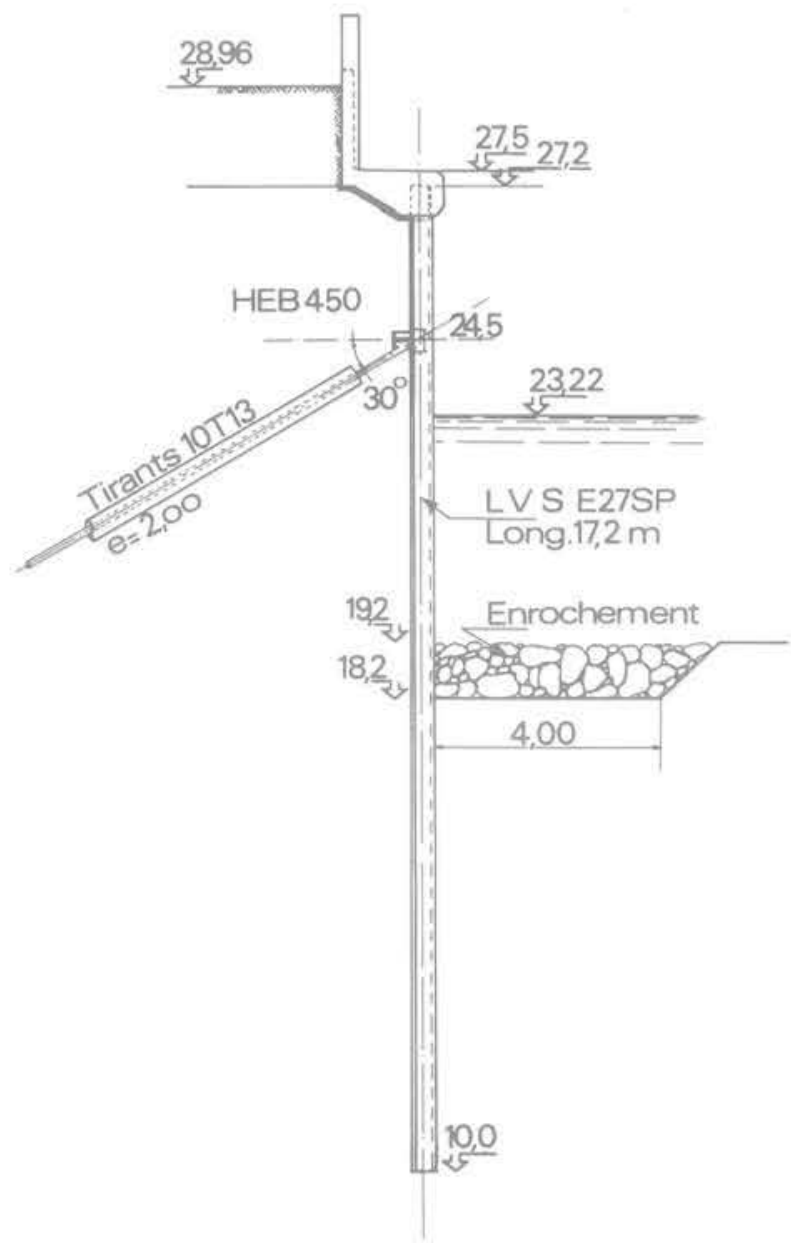

Fig. 1 Coupe transversale du rideau

Toutes les cotes sont exprimées en m NGF (Nivellement Général de la France) 


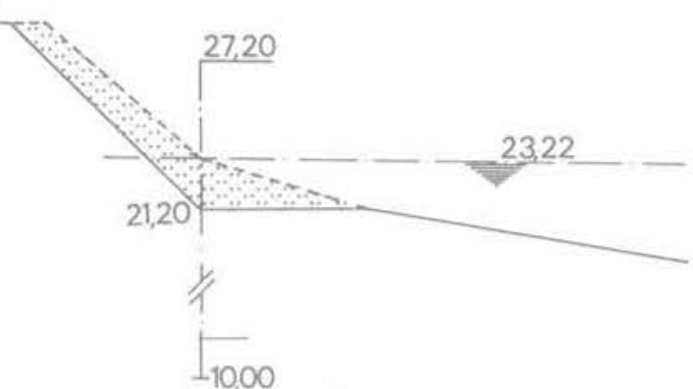

PHASE 1

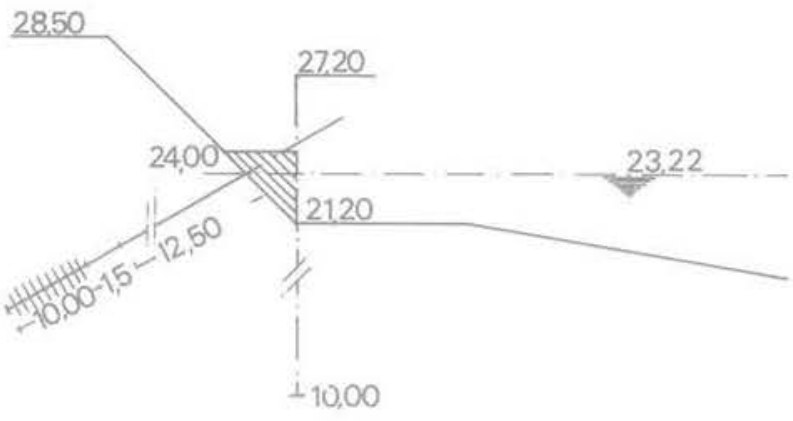

PHASE II

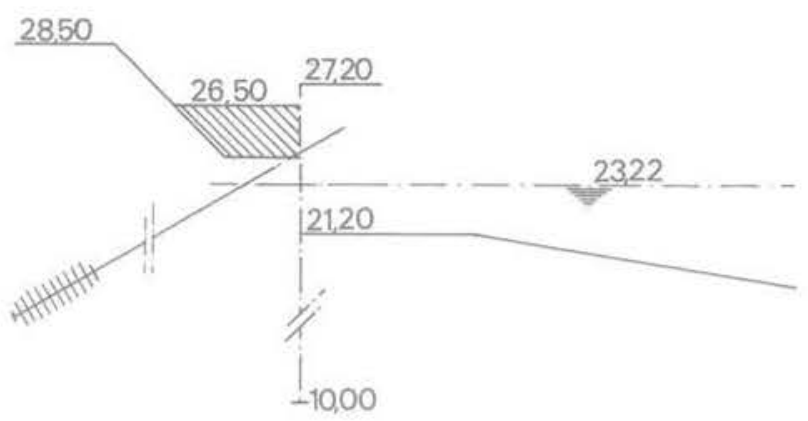

PHASE III

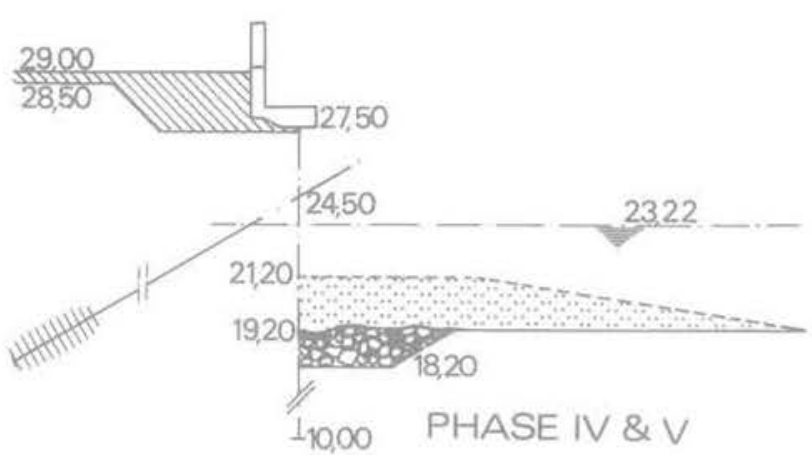

Fig. 2 Principales phases de travaux

1 Prédragage - battage

2 Remblaiement - mise en tension partielle des

tirants

3 Suite du remblaiement - mise en tension défini-

tive des tirants

4-5 Fin du remblaiement - dragage

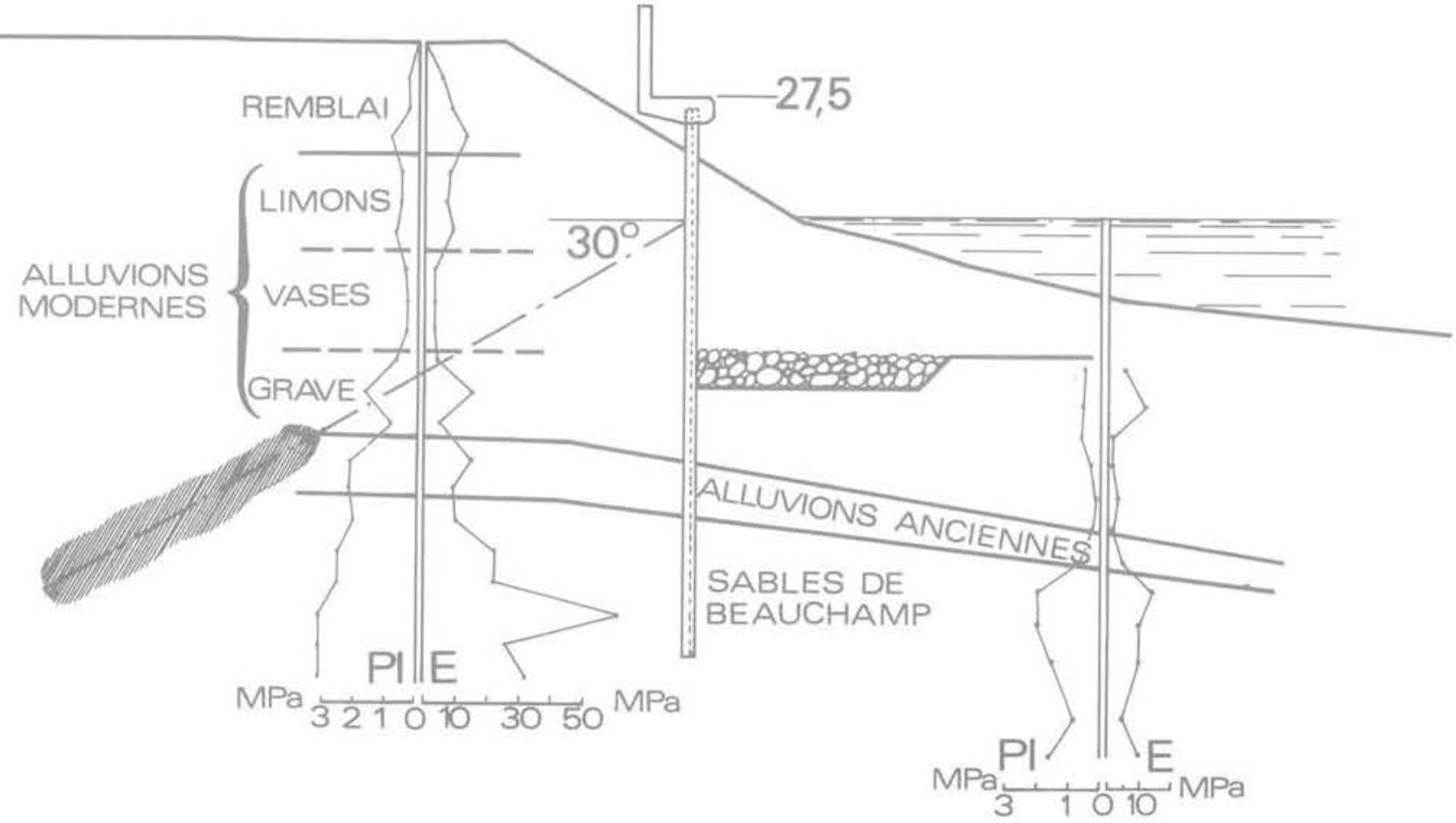

Fig. 3 Profil géotechnique au droit du rideau 


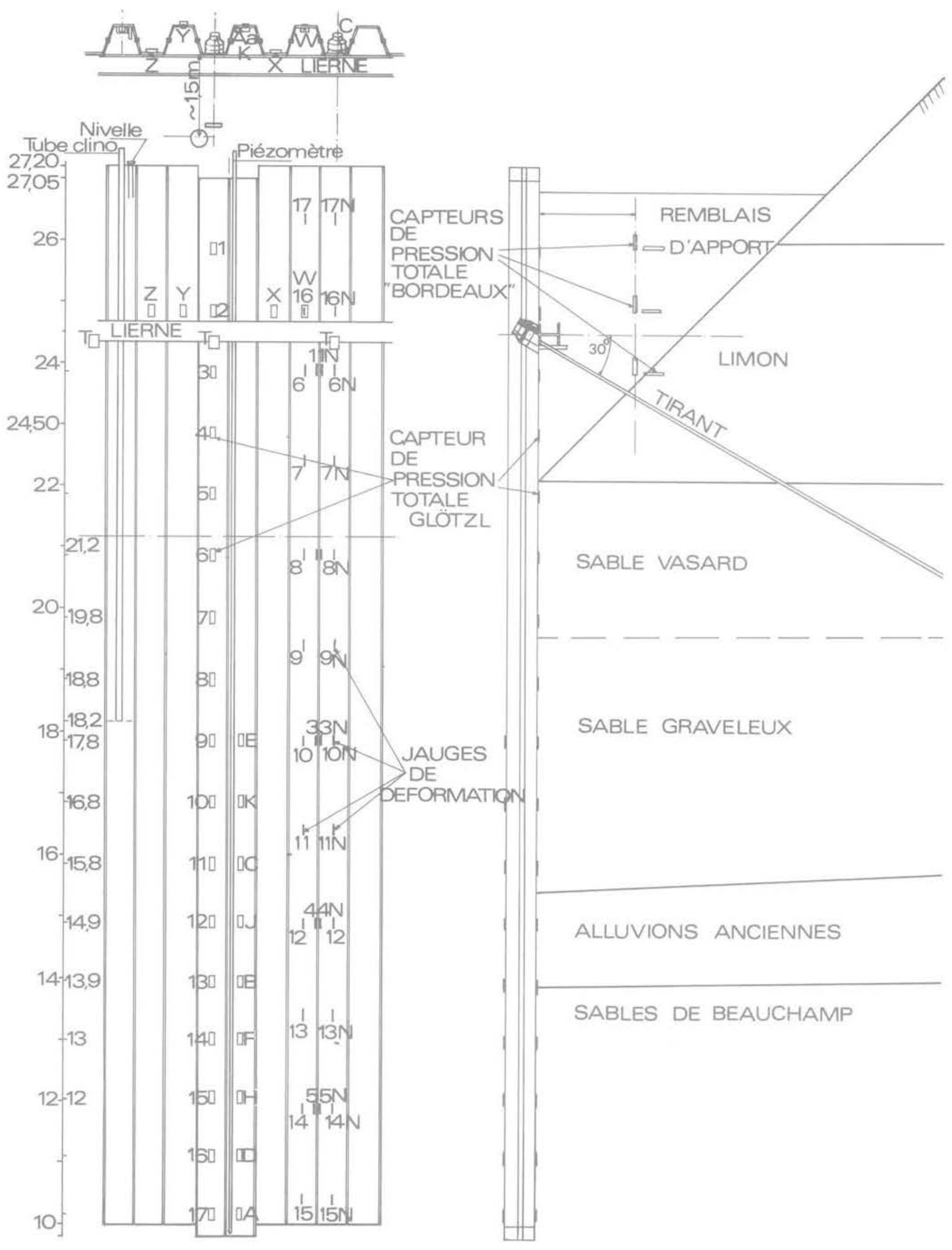

Fig. 4 Equipement de palplanches

- vues de dessus et élévation

- coupe transversale 


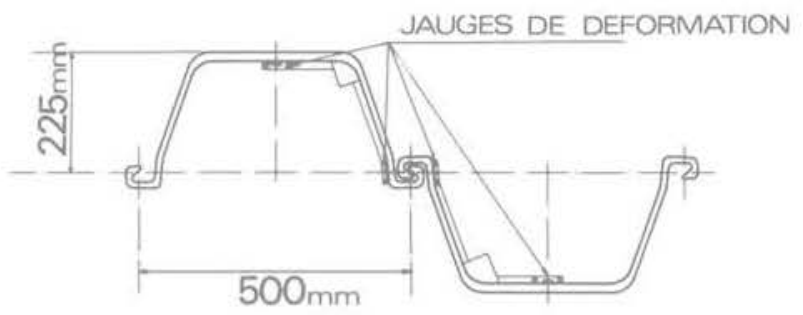

Fig. 5 Section d'une paire de palplanches précisant la position des jauges de déformation

\subsection{Les phases de travaux}

Afin de limiter les déplacements de l'ouvrage, la construction comporte une alternance de remblaiements à l'arrière du rideau et de mises en tension successives des-tirants. Le dragage en Seine intervient en dernier lieu.

Les principales phases de travaux sont les suivantes (cf. fig, 2).

- Battage des palplanches après prédragage et réglage du talus à $1 / 1$.

- Mise en tension partielle des tirants après une première phase de remblaiement à $+24 \mathrm{~m}$ NGF.

- Remblaiement par étapes jusqu'à $+26,50 \mathrm{~m} \mathrm{NGF}$ et mise en tension définitive à $750 \mathrm{kN}$.

- Construction du mur de couronnement et remblaiement à la cote $+29,00 \mathrm{~m} \mathrm{NGF}$.

-Dragage à la cote $+19,20 \mathrm{~m}$ NGF.

\subsection{Le site - ses caractéristiques géotechniques}

La coupe des terrains, déterminée par 1 sondage carotté et deux forages pressiométriques, est la suivante (fig. 3 ).

- $29 \mathrm{~m}$ à 26 m NGF Remblais Sablo-limoneux

- $26 \mathrm{~m}$ à 15,50 m NGF Alluvions modernes : Limons sur 3 à $4 \mathrm{~m}\left(\mathrm{c}=0\right.$ à $\left.40 \mathrm{kPa}-\varphi=30^{\circ}\right)$

Sables fins coquilliers vaseux sur 3 mètres $p_{1}<400 \mathrm{kPa}$ $c=0 \varphi=32$ à $39^{\circ}$

Sables grossiers sur 4 mètres $p_{1}=600$ à $1000 \mathrm{kPa}$

- 15,50 m à $14 \mathrm{~m}$ NGF Alluvions anciennes sablo-graveleuses $\left(\varphi=37^{\circ}\right)$

- en dessous de 14 m NGF Sables de Beauchamp: Sables fins argileux très compacts, avec quelques niveaux grésifiés $\mathrm{p}_{1}>2 \mathrm{MPa} \quad c^{\prime}=50 \mathrm{kPa} \quad \varphi=30$ aे $35^{\circ}$

Les caractéristiques de cisaillement indiquées sont des moyennes d'essais à la boite de Casagrande. On notera en particulier les angles de frottement élevés mesurés dans des alluvions de nature très médiocre.

Le matériau de remblaiement à l'arrière de rideau était un tout venant respectant les critères suivants :

- Passant à $80 \mu<5 \%$

- Equivalent de sable E.S. > 35

- Angle de frottement interne $\varphi>35^{\circ}$

- hors nappe, compactage par tranches de 20 à $30 \mathrm{~cm}$, densité supérieure $95 \%$ de l'optimum proctor modifié.

\section{L'équipement des palplanches}

Cet équipement a été réalisé pour une grande part en atelier, puis complété sur chantier après le battage.

\subsection{Le programme d'équipement}

L'équipement complet des palplanches est présenté sur la figure $n^{\circ} 4$ et décrit ci-après:

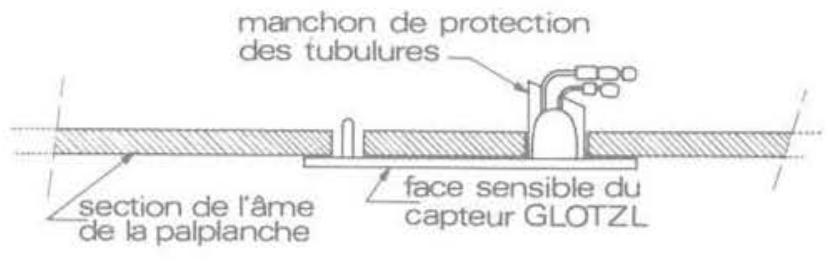

Fig. 6 Schéma de pose des capteurs de pression totale

$1^{\circ}$ paire de palplanches:

mesure des pressions des terres

L'équipement comprend:

-17 capteurs de pression totale Glötzl, espacés de 1 mètre pour mesurer les poussées.

- 9 capteurs, également espacés de 1 mètre pour mesurer les butées.

$2^{\circ}$ paire de palplanches:

mesure des contraintes dans les palplanches

-34 jauges de contraintes «AILTECH » ont été soudées selon la répartition suivante:

-5 sections transversales espacées de 3 mètres, équipées de 4 jauges: 1 sur chaque âme et 2 de part et d'autre de la serrure (fig. 5).

-7 autres sections équipées seulement de 2 jauges sur les âmes.

\section{$3^{e}$ paire de palplanches:}

\section{mesure des déformées}

- Un tube inclinométrique carré a été fixé sur une palplanche puis protégé par un capot métallique soudé.

Sur cette même palplanche, après battage, une console a été fixée en tête, afin de faire des mesures de rotation à l'aide d'une nivelle à bulle.

\section{Etude complémentaire des pressions}

à l'arrière du rideau

Après la $1^{\text {re }}$ phase de remblaiement et la pose de la lierne, 4 capteurs Glötzl ont été posés juste au-dessus de cette lierne dont 2 en "bosse " et 2 en "creux", afin d'étudier la répartition horizontale des pressions au niveau des tirants.

\section{Equipement des tirants}

Deux cales dynamométriques Glötzl ont été placées sur deux tirants consécutifs intéressant la zone équipée. Les techniques de pose des divers appareillages, exposées en détail dans un autre document (Gigan - 1979) ont impliqué parfois des travaux fort minutieux. Ainsi, la fixation des capteurs de pression totale a nécessité le percement préalable de 2 trous à travers la palplanche (fig. 6). Tous les fils de mesure ainsi que les jauges étaient protégés par des capots métalliques et noyés dans un produit souple.

Des mesures de la flèche des palplanches posées sur appuis simples ont permis de vérifier que cet équipement ne modifiait pas de manière sensible leur inertie.

\subsection{Comportement des appareils lors du battage}

La mise en œuvre des palplanches sur le chantier a été émaillée de divers incidents, liés essentiellement à une fixation insuffisante des capots de protection des appareillages.

Le plus facheux a été la destruction de tubulures en plastique des capteurs de pression totale lors d'une tentative 


\begin{tabular}{|c|c|c|c|}
\hline Date & Phase de travaux & $\begin{array}{c}\text { Déplacement partiel } \\
-=\text { vers la terre } \\
+=\text { vers la Seine }\end{array}$ & Déplacement cumulé \\
\hline 03.75 & Remblaiement partiel & non mesuré & $+0,9 \mathrm{~cm}^{*}$ \\
\hline 2.04 .75 & $\begin{array}{l}\text { Mise en tension } \\
\text { partielle }(150 \mathrm{kN}) \\
\text { des tirants } n^{\circ} 150 \\
\text { à } 153\end{array}$ & $-3,9 \mathrm{~cm}$ & $-3,0 \mathrm{~cm}$ \\
\hline $\begin{array}{l}\text { du } 2.04 \text { au } \\
7.04\end{array}$ & $\begin{array}{l}\text { remblaiement jusqu'à } \\
+26,50\end{array}$ & $+1,9 \mathrm{~cm}$ & $-1,1 \mathrm{~cm}$ \\
\hline 7.04 & $\begin{array}{l}\text { mise en tension à } \\
750 \mathrm{kN} \text { des tirants } \\
N^{0} 151 \text { et } 152\end{array}$ & $-1,6 \mathrm{~cm}$ & $-2.7 \mathrm{~cm}$ \\
\hline 8.04 & $\begin{array}{l}\text { Mise en tension à } \\
750 \mathrm{kN} \text { des tirants } \\
\text { No } 153 \text { et suivants }\end{array}$ & $-1,4 \mathrm{~cm}$ & $-4.1 \mathrm{~cm}$ \\
\hline 8.75 & $\begin{array}{l}\text { Dragage entre }+21,20 \\
\text { et } 19,20\end{array}$ & $+0,4 \mathrm{~cm}$ & \\
\hline
\end{tabular}

* valeur estimée à partir des relevés effectués sur d'autres profils.

Tableau 1 Déplacements de la tête du rideau

de ressoudage des capots. Leur remise en état a nécessité l'arrachage d'une palplanche en cours de battage. Le déplacement d'un élément de tube inclinomètre a empêché les mesures angulaires en partie inférieure.

Par contre, il faut souligner le bon fanctionnement de toutes les jauges malgré un battage énergique au mouton Delmag D.12.

\section{Mesure des déformées des palplanches}

\subsection{Mesure des déplacements de la tête du rideau}

Les déplacements de 3 tiges métalliques, soudées en tête de palplanches à l'aplomb des tirants $n^{\circ} 150-152$ et 153 ont été suivis par nivellement à l'aide d'un niveau N 2 . Les déplacements mesurés (moyenne des 3 valeurs) sont indiqués dans le tableau 1

On constate que la $1^{\text {re }}$ phase de remblaiement provoque un faible déplacement du rideau vers la Seine, mais que pour toutes les phases ultérieures de travaux, le rideau " rentre " dans le remblai.

\subsection{Déformées des palplanches}

Les déformées successives des palplanches sont déduites des relevés inclinométriques. Les mesures angulaires, permettant de lire le $1 / 100^{\circ}$ de degré ont été confirmées par les valeurs de rotations en tête données par la nivelle à bulle d'une précision bien plus grande (quelques secondes d'arc).

En l'absence de référence par rapport au pied du rideau par suite de la détérioration de la base du tube inclinométrique, ces déformées ont été "calées» à partir des mesures de déplacement en tête.

Les déformées du rideau sont tracées (entre +27 et $+18 \mathrm{~m} \mathrm{NGFl}$, sur la figure 7.

\section{Les mesures des contraintes dans les palplanches} - détermination des moments fléchissants

\subsection{Diagrammes de contraintes}

Les mesures d'allongement fournies par les jauges ont été converties en valeurs de contraintes.

$\sigma=E \frac{\Delta l}{l}$ avec $E=2,110^{8} \mathrm{kN} / \mathrm{m}^{2}$

Les diagrammes (cf fig. 8) montrent que :

- Des contraintes de signe opposé, sont obtenues de part et d'autre des serrures. Le rideau ne doit donc pas être considéré comme une poutre monolithique, au contraire, les deux palplanches d'une paire semblent se comporter de manière indépendante, par suite d'un glissement relatif dans les serrures, ce résultat étant surtout net pour les sections situées à $+21 \mathrm{~m}$ et $+18 \mathrm{~m} \mathrm{NGF}$. II faut rappeler que les palplanches étaient mises en fiche individuellement, qu'elles n'étaient ni pincées, ni soudées.

$-\mathrm{A}$ la base du rideau (sections situées à $+15 \mathrm{~m}$ et +12 m NGF), on mesure des contraintes de traction dans la palplanche extérieure (côté Seine) et des contraintes de compression dans la palplanche intérieure (côté terre).

\subsection{Détermination des moments fléchissants}

La détermiliation des moments fléchissants et des efforts normaux à partir des mesures de contraintes est relativement aisée dans les deux cas extrêmes: (cf fig. 9)

a) - Rideau monolithique

b) - Palplanches indépendantes

En fait, il est probable que le comportement du rideau est intermédiaire entre ces deux cas (glissement partiellement empêché dans les serrures), mais le calcul devient alors beaucoup plus difficile. 


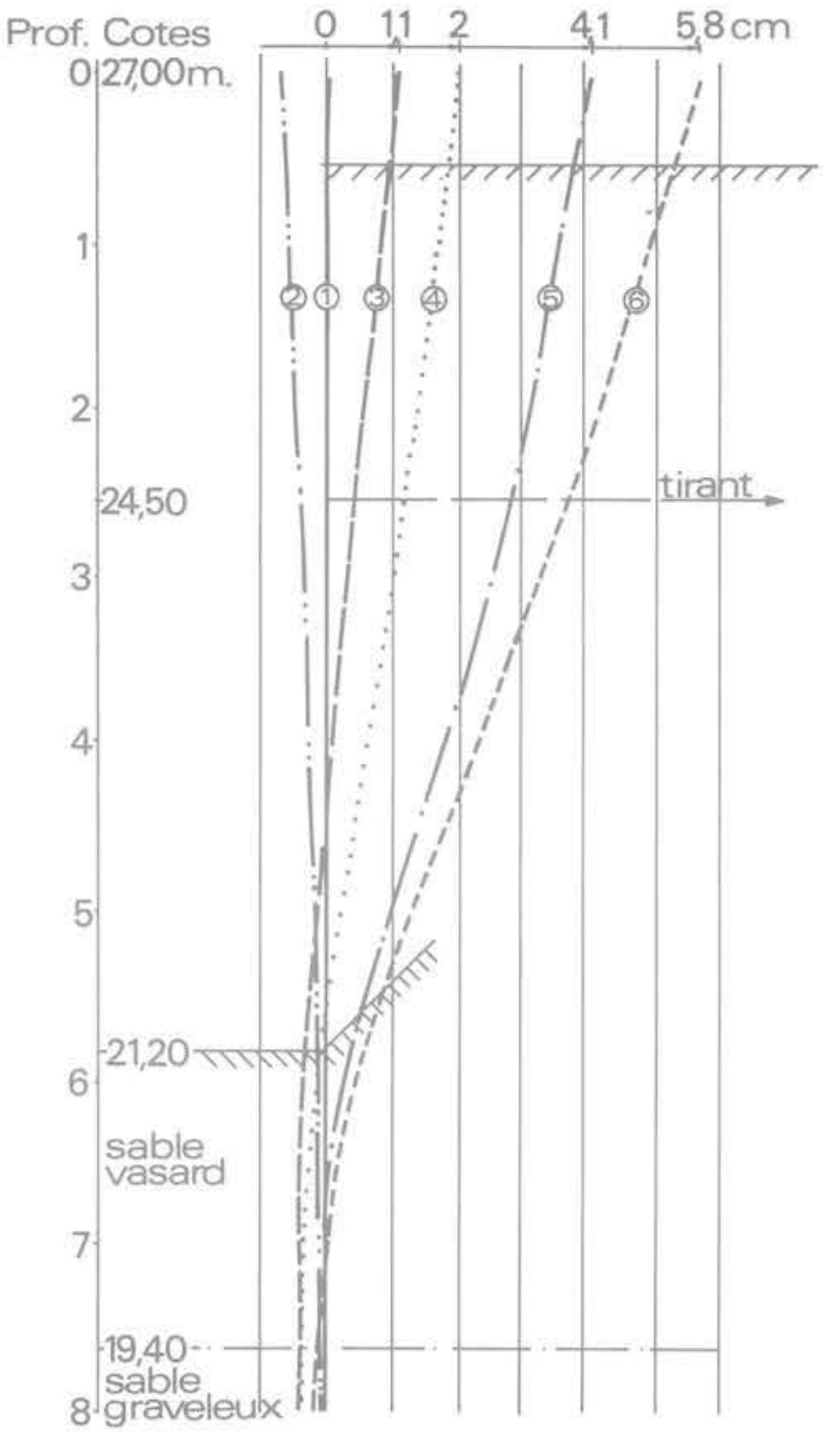

Fig. 7 Déformées de la palplanche déduites des mesures inclinométriques

- mesure n 1 28/02/75 Battage

- mesure ${ }^{\circ} 2$ 06/03/75 Remblai à + 24 NGF

(mesure de référence pour l'inclinomètre)

- mesure $n^{\circ} 3 \quad 04 / 04 / 75$ Après mise en tension partiel-

le à $150 \mathrm{kN}$ et remblaiement à $+26,50$

- mesure n $n^{\circ}$ 07/04/75 Après blocage à $750 \mathrm{kN}$ des tirants 151 et 152

- mesure $n^{\circ} 5$ 08/04/75 Après blocage à $750 \mathrm{kN}$ de la ligne de tirants

- mesure $n^{\circ} 6 \quad 23 / 04 / 75 \quad 17$ jours après la mise en tension

Au vu des diagrammes de contraintes, l'hypothèse des palplanches indépendantes s'avère ici beaucoup plus représentative du comportement réel du rideau et a été retenue dans les calculs qui consistent alors, en admettant un état de flexion composée, à calculer un effort normal et un moment de flexion pour chaque palplanche d'une paire. (figures 10 et 11 )

\subsubsection{Commentaires sur les diagrammes d'effort normaux (fig. 10)}

\section{Palplanche côté terre (avec tirant)}

Sous le tirant, les efforts normaux de compression augmentent jusque vers $+18 \mathrm{~m}$ NGF et sont supérieurs à la composante verticale de l'effort d'ancrage. II faudrait donc admettre une obliquité vers le bas des efforts de poussées. Par contre, l'effort normal décroít rapidement en dessous de +15 m NGF pour s'annuler en pied de palplanche.

Palplanche côté Seine (sans tirant)

Dans cette palplanche, les efforts mesurés sont des tractions, qui augmentent au fur et à mesure des phases de travaux. L'effort reste à peu près constant entre $+24 \mathrm{~m}$ et $+15 \mathrm{~m} \mathrm{NGF}$ et décroit en dessous.

Nous n'avons pas trouvé d'explication réellement satisfaisante de ce phénomène.

\section{Diagramme résultant}

Avant la mise en prétension du tirant, on observe un effort résultant de traction. Par la suite, le rideau est comprimé, l'effort étant maximal vers $+18 \mathrm{~m} \mathrm{NGF}$. Toutefois à $+21 \mathrm{~m} \mathrm{NGF}$ l'effort de compression est inférieur à la composante verticale de l'effort d'ancrage.

\subsubsection{Commentaires sur les diagrammes de} moments fléchissants (fig. 11)

Les moments ne diffèrent pas de plus de 10 à $15 \%$ entre les deux palplanches, qui ont donc un comportement sensiblement identique.

Après la mise en tension des tirants, on trouve un moment négatif à $+21 \mathrm{~m}$ et $+18 \mathrm{~m} \mathrm{NGF}$, et positif à $+15 \mathrm{~m}$ et $+12 \mathrm{~m} \mathrm{NGF}$, ce qui caractérise bien un comportement du type rideau encastré.

Le point de moment nul devrait se situer entre $+15 \mathrm{~m}$ et $+16 \mathrm{~m} \mathrm{NGF}$.

Le moment fléchissant maximal, atteint vers $+20 \mathrm{~m} \mathrm{NGF}$ vaut environ $260 \mathrm{mkN} / \mathrm{ml}$ après la mise en tension, on peut constater que cette valeur est très voisine de celle obtenue en construisant la courbe des moments à partir de mesures de pressions totales. (cf fig. 15)

Par contre les moments obtenus pour la partie en fiche restent faibles.

\section{Les mesures de pression des terres}

5.1 Diagrammes de pressions mesurées pour les principales phases de travaux - détermination des coefficients de poussée et de butée

Nous avons retenu 3 des diagrammes les plus significatifs (cf fig 12).

le $28.02 .75: 3$ jours après battage du rideau

le 07.04.75: Après mise en tension partielle des tirants $(150 \mathrm{kN})$ et remblaiement à $+26,5 \mathrm{~m}$ NGF.

Le 22.05.75:40 jours après mise en tension définitive des tirants $(750 \mathrm{kN})$ - même cote de remblaiement.

Un piézomètre situé à l'arrière du rideau a confirmé que le niveau de la nappe y était identique à celui de la Seine. Ceci nous a permis d'exprimer les résultats en pressions effectives.

\section{Pressions des terres après battage -} diagramme du 28.02.75

Après le battage, les poussées restent très faibles dans les alluvions. Si, compte tenu du profil de la berge, il est difficile de définir un coefficient de poussée initial, on remarque que les pressions effectives sont pratiquement nulles entre $+18 \mathrm{~m}$ et $+16 \mathrm{~m} \mathrm{NGF}$, à la base des alluvions modernes, pourtant sableuses.

Le matériau semble avoir un comportement cohérent, la cohésion ou "pseudo-cohésion » ayant pu d'ailleurs être provoquée par les vibrations des palplanches lors du battage.

Dans les Sables de Beauchamp, on constate une bonne symétrie des diagrammes de pression, les pics pouvant correspondre à des niveaux plus résistants ou grésifiés. Les pressions effectives horizontales sont généralement supérieures aux pressions verticales calculées (le rapport $\frac{\sigma_{h}^{\prime}}{\sigma_{v}^{\prime}}$ varie entre 0,6 et 3,5$)$. 

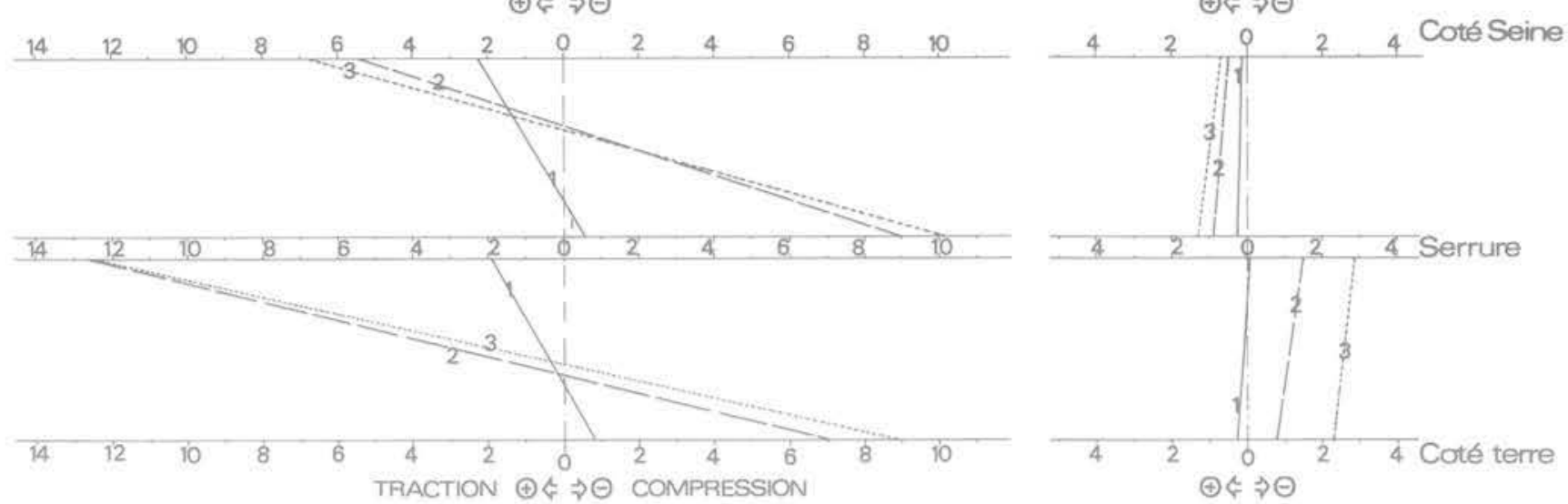

Fig. 8 Diagrammes de contraintes dans les palplanches

$128 / 03 / 751$ mois après battage - remblaiement à +24 NGF

$207 / 04 / 75$ Remblaiement $\grave{a}+26,5 \mathrm{NGF}$ - avant mise en tension

$307 / 04 / 75$ Remblaiement à $+26,5$ NGF-après mise en tension

PALPLANCHE AVEC TIRANT

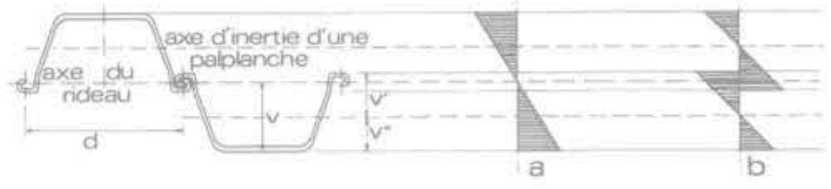

Fig. 9 Schéma indiquant les contraintes de flexion dans le rideau

a rideau monolithique

b palplanches indépendantes (glissement dans les ser. rures)

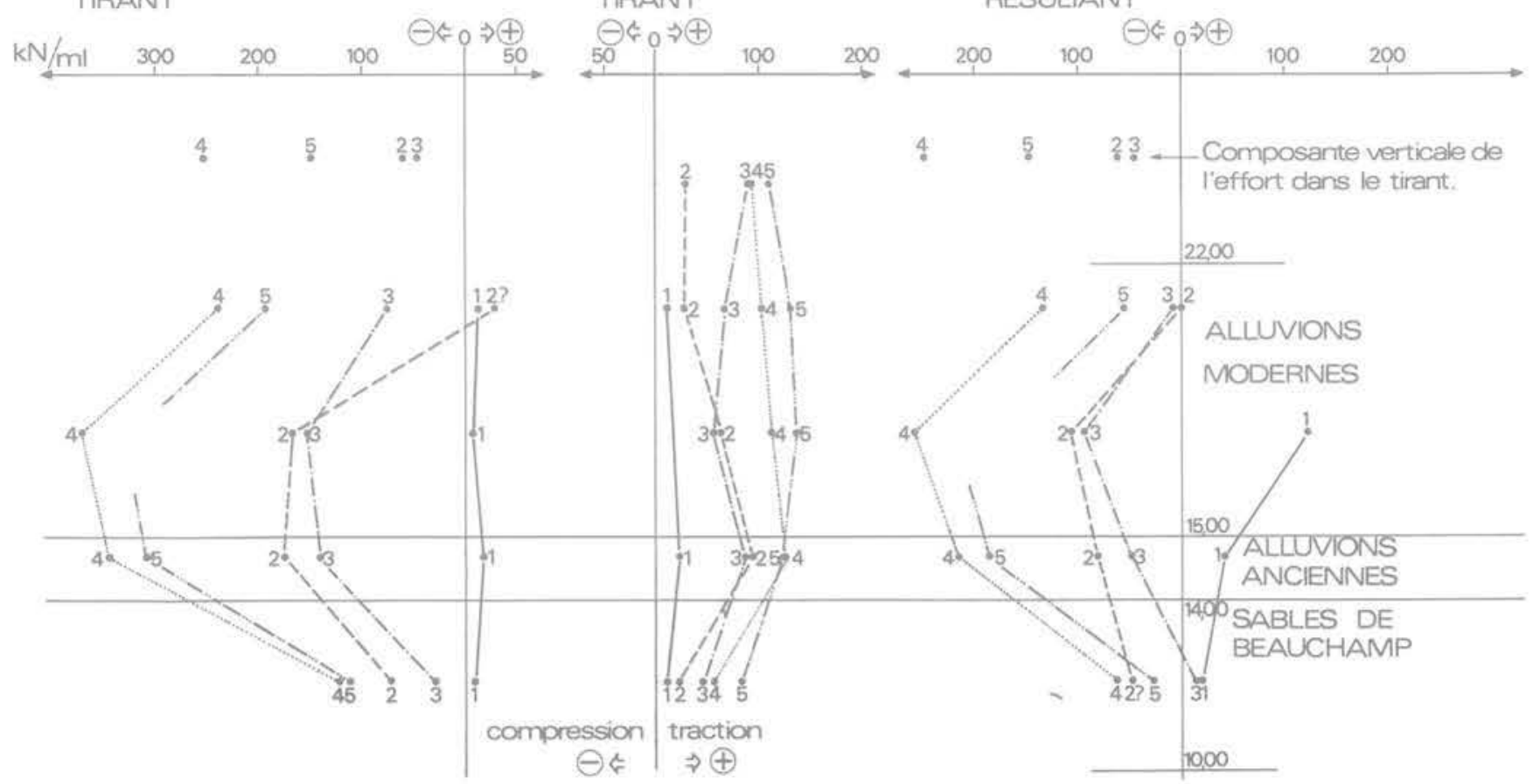

Fig. 10 Diagrammes des efforts normaux dans les palplanches 
M1

palplanche

avec tirant
MOMENT FLECHISSANT

$\mathrm{M} 2$

palplanche

sans tirant
$\mathrm{M}$

diagramme

résultant

$(\mathrm{M}=\mathrm{M} 1+\mathrm{M} 2)$

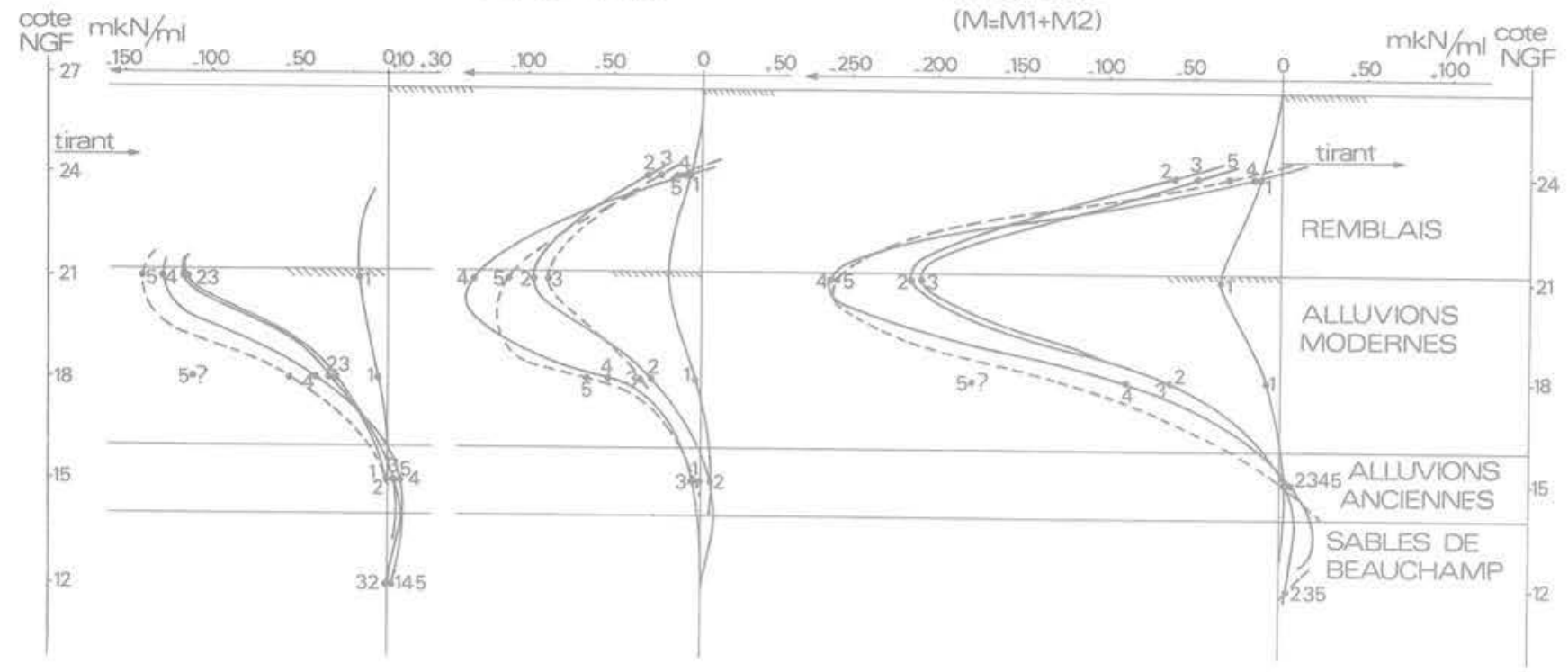

Fig. 11 Diagrammes des moments fléchissants déduits des mesures par jauges (interprétation en palplanches indépendantes)

$\begin{array}{lllll}1 & 28 / 03 / 75 & \text { Remblaiement à }+24 N G F & 4 & 07 / 04 / 75 \\ 2 & 02 / 04 / 75 & \text { Mise en prés mise en tension à } 750 \mathrm{kN} \\ 3 & 07 / 04 / 75 & \text { Avant mise en tension à } 150 \mathrm{kN} & 5 & 22 / 05 / 75 \text {. }\end{array}$

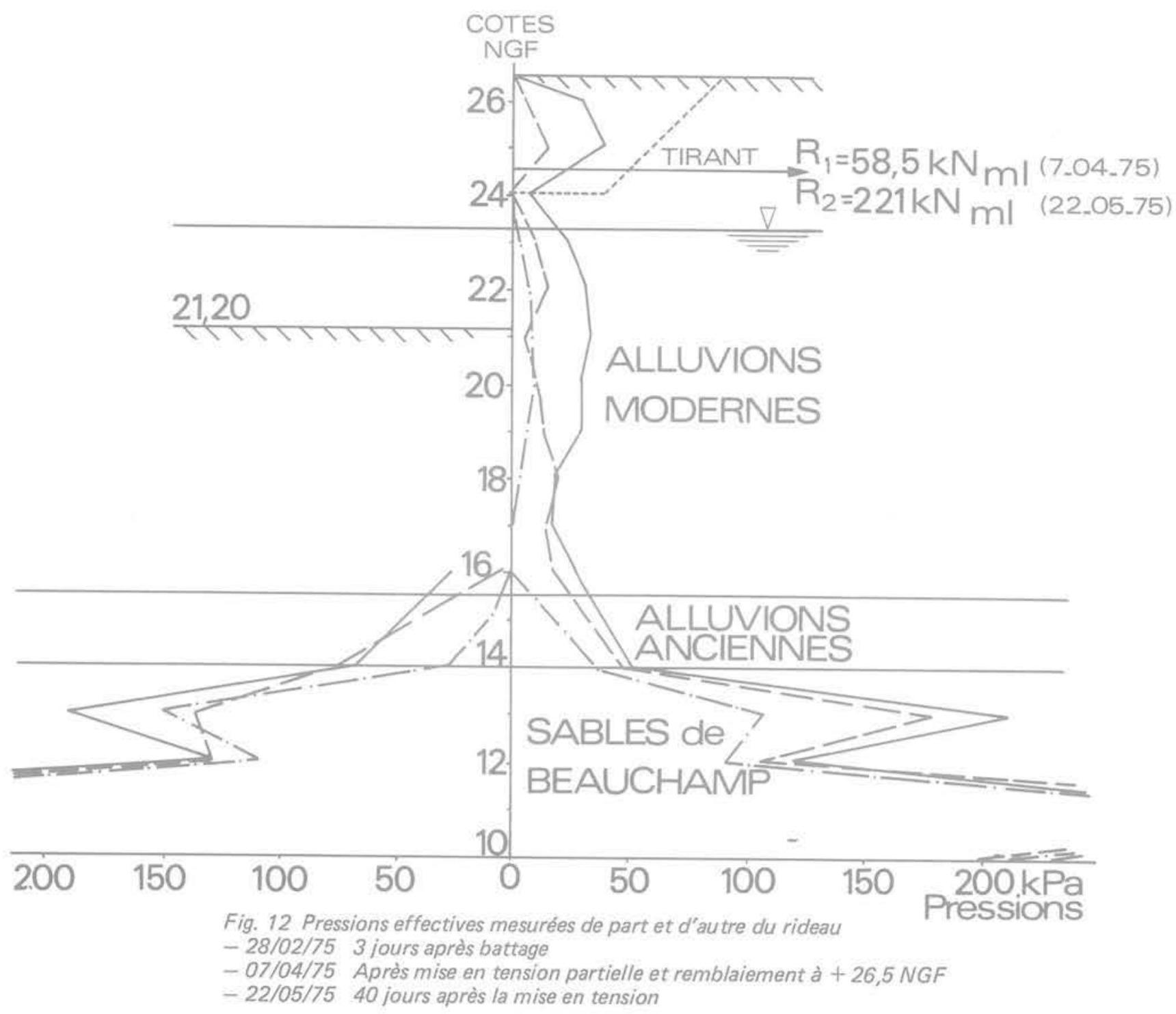




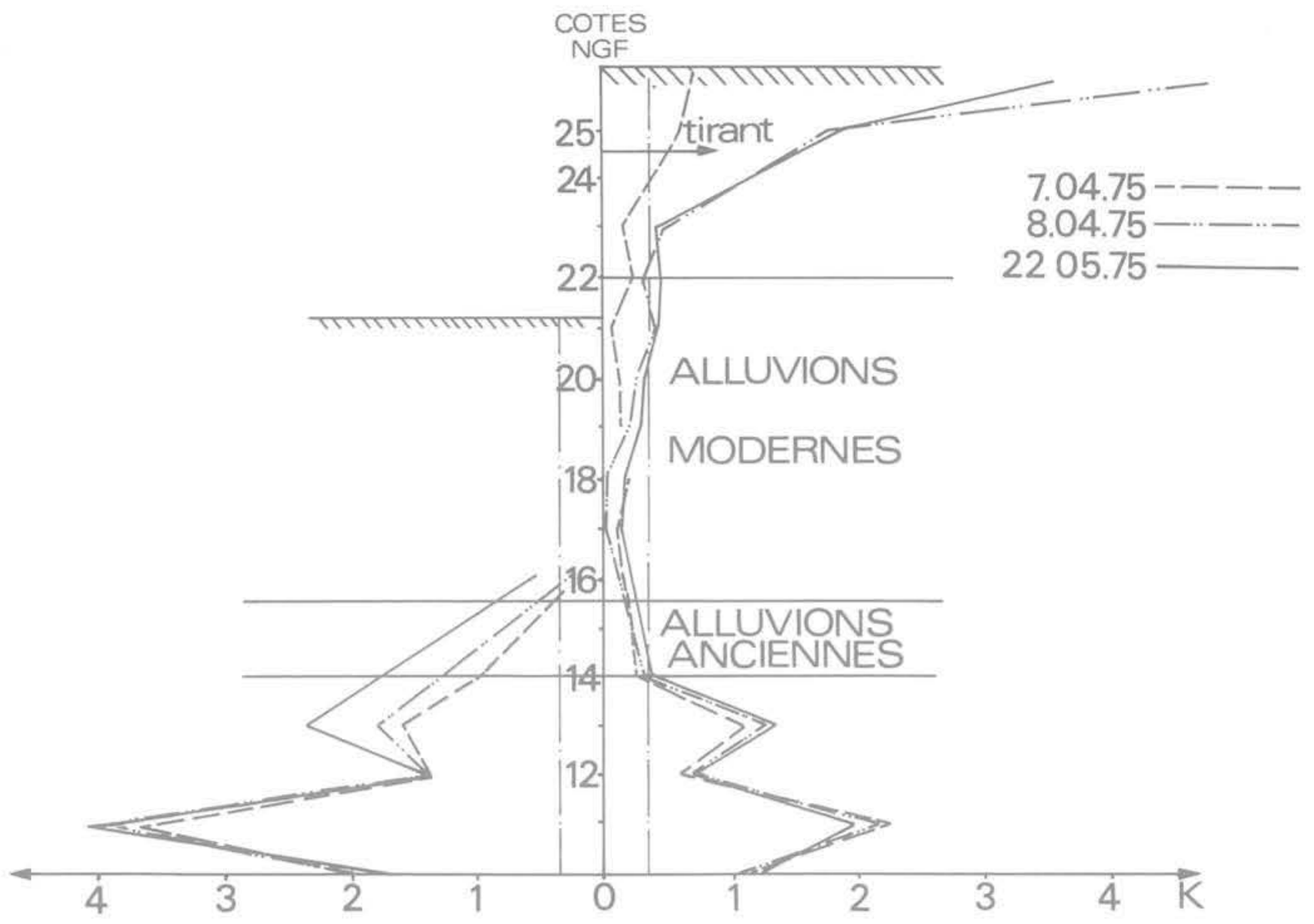

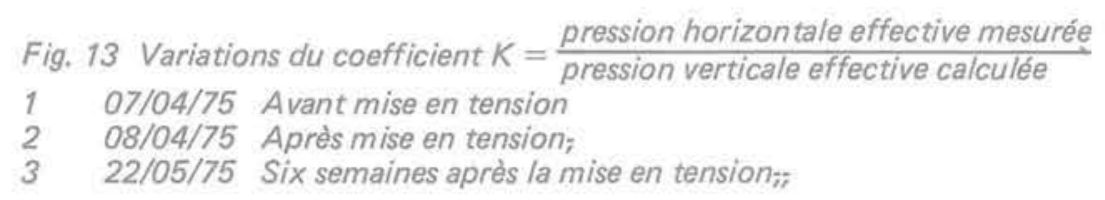

Pression des terres après la prétension des tirants et le remblaiement á + 26,50 - mesure du 07.04.75

Le rapport

$K=\frac{\sigma_{h}^{\prime} \text { pression effective horizontale mesurée }}{\sigma_{v}^{\prime} \text { pression effective verticale calculée }}$ prend les valeurs suivantes: (fig. 13).

$\mathrm{K}=0,6$ à 0,7 dans les remblais compactés par tranches au dessus du tirant

$K=0,1$ à 0,2 dans les remblais noyés et les alluvions modernes côté terre

$K=0,3$ à 3,5 dans les sables de Beauchamp.

On note surtout les faibles coefficients de poussée obtenus dans les alluvions et une légère augmentation des pressions, aussi bien côté terre que côté Seine dans les Sables de Beauchamp après le remblaiement.

Effet de la mise en tension des tirants - comparaison des résultats du 7.04.75 et du 22.05.75

La mise en tension du tirant provoque une nette augmen- tation des pressions non seulement au dessus de celui-ci. mais aussi en dessous jusqu'à +19 m NGF. Ce résultat est tout à fait conforme aux mesures des déformées, indiquant un enfoncement vers le massif de toute la partie hors fiche du rideau.

Par contre, on note une légère diminution des poussées à $+18 \mathrm{~m}$ et $+19 \mathrm{~m} \mathrm{NGF}$

Dans les Sables de Beauchamp, on constate encore un accroissement simultané des pressions côté Seine et côté terre.

Les coefficients de poussée deviennent:

$\mathrm{K}=4$ et

$K=1,6$ dans les remblais au dessus du tirant

$\mathrm{K}=0,3$ à 0,5 dans les remblais et alluvions jusqu'à $+19 \mathrm{~m} \mathrm{NGF}$

$\mathrm{K}=0,15$ aे 0,22 entre $+18 \mathrm{~m}$ et $+16 \mathrm{~m} \mathrm{NGF}$ $K=0,7$ à 4 dans les Sables de Beauchamp.

Après la mise en tension, les valeurs des pressions ont continué d'évoluer pendant plusieurs mois. Ainisi sur la figure 13 sont indiquées les valeurs du coefficient $\mathrm{K}$ six semaines après le blocage des tirants. 


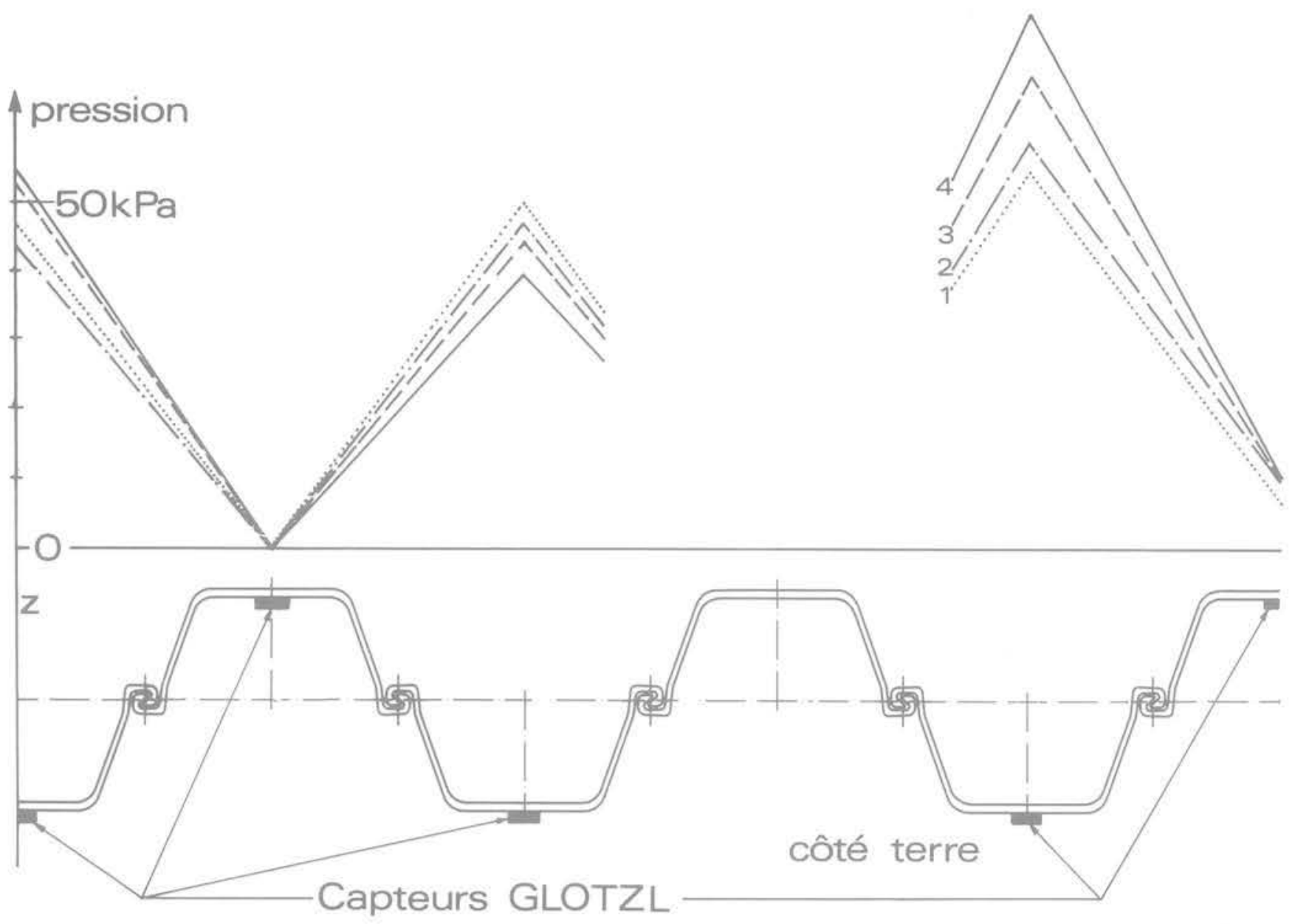

Fig. 14 Répartition des pressions entre les "creux》 et les "bosses》 des palplanches

$\begin{array}{ll}1 & 07 / 04 / 75 \\ 2 & 15 / 04 / 75 \\ 3 & 22 / 05 / 75 \\ 4 & 18 / 07 / 75\end{array}$

Distribution des pressions des terres entre les creux et les bosses des palplanches (fig. 14)

Sur un axe horizontal, situé juste au dessus de la lierne et au niveau des tirants, des capteurs avaient été placés alternativement sur les "bosses" et dans les creux des palplanches.

Dans les creux des palplanches, les valeurs des pressions mesurées restent très faibles (inférieures à $10 \mathrm{kPa}$ ) pour toutes les phases de travaux. Un effet de voûte semble se créer dans le remblai, favorisé certainement par un défaut de compactage dans le creux des palpianches.

On peut donc se demander dans quelle mesure des pressions mesurées sur les « bosses 》 nous fournissent un profil des pressions moyennes appliquées au rideau. A ce titre, la mesure des pressions au sein du terrain, sur une verticale distante de 0,5 à $1 \mathrm{~m}$ du rideau aurait été préférable.

\subsection{Equations d'équilibre du rideau}

Compte tenu des mauvais fonctionnements de certains capteurs, de modifications des valeurs de linertie de quelques cellules constatées après le battage et des écarts

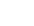




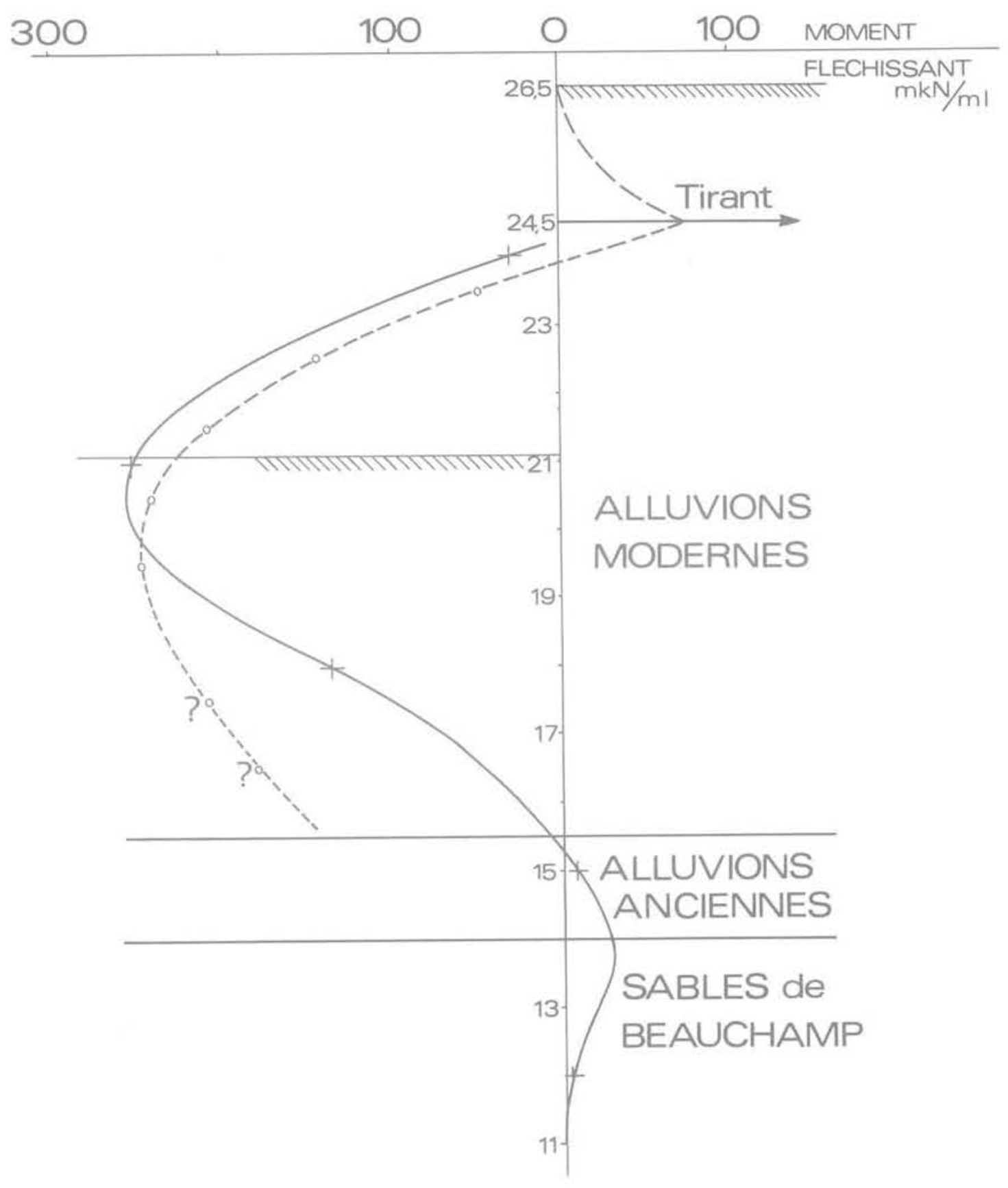

Fig. 15 Courbes de moments fléchissants:

déduite des mesures de pression totale déduite des mesures de contraintes (jauges)

- L'effort d'ancrage est à peu près compensé par l'accroissement des pressions à l'arrière du rideau dans la partie hors fiche:

Variation des pressions totales à l'arrière du rideau entre $+19 \mathrm{~m}$ et $+27 \mathrm{~m} \mathrm{NGF}$, due à la mise en tension

Variation de la composante horizontale de l'effort dans le tirant

$170 \mathrm{kN} / \mathrm{ml}$

$163 \mathrm{kN} / \mathrm{ml}$

- Ces réactions ont leur centre de gravité situé en dessous du point d'ancrage (22,80 m NGF contre 24,50 m NGF). Ainsi le couple formé par l'effort d'ancrage et les réactions de butée partielle du sol a pour effet d'augmenter le moment fléchissant sous le tirant.
5.4 Comparaison des moments fléchissants déduits respectivement des mesures de pression des terres et des contraintes dans la palplanche

Pour la partie supérieure du rideau, la courbe des moments fléchissants calculés à partir des mesures de pression est tracée sur la figure 15. Au dessus de $+19 \mathrm{~m}$ NGF, on obtient une concordance satisfaisante avec la courbe déduite des mesures de déformation. Par contre, la concordance n'est plus bonne en-dessous, où les pressions sont moins bien connues. 


\section{Evolution de la tension des tirants précontraints}

Les deux tirants équipés de cales dynamométriques ont été mis en tension simultanément. Lors du blocage, les valeurs indiquées par les cales étaient de $780 \mathrm{kN}$ et $710 \mathrm{kN}$ (valeur théorique de $750 \mathrm{kN}$ )

Par la suite, la mise en tension des tirants adjacents ayant provoqué un enfoncement de la lierne et de l'ensemble du rideau, on a constaté une chute brutale des tensions des deux premiers tirants (respectivement à 640 et $560 \mathrm{kN}$ ) qui se sont stabilisées par la suite (fig. 16).

II faut noter que ces fortes chutes de tension, liées au processus particulier de mise en tension adopté au niveau des palplanches expérimentales, n'ont pas été observées sur les autres tirants de l'ouvrage.

7 Calcul du rideau par des méthodes élastoplastiques - comparaison des résultats du calcul avec les valeurs expérimentales - Ajustement des paramètres

\subsection{But du calcul}

Détermination des principaux paramètres

Le dépouillement et l'interprétation des mesures effectuées tout au long de la construction du rideau ont fourni :

- les déformées du rideau

- les diagrammes de moment fléchissant

- les diagrammes de pression des terres

- les efforts d'ancrage dans les tirants

Nous avons vu que ces résultats sont connus avec une précision variable et notamment que les moments fléchissants ont été calculés en tenant compte d'un glissement dans les serrures, mis en évidence par les jauges de déformation.

Le dimensionnement initial du rideau avait été réalisé par une méthode élastoplastique, tenant compte des phases intermédiaires de travaux.

Ces méthodes se sont largement développées ces dernières années pour le calcul des parois moulées et rideaux ancrés par tirants actifs, elles présentent l'avantage de permettre une simulation aisée des mises en tension de tirants, ce qui n'est pas le cas avec les méthodes classiques aux états limites.

Nous avons réutilisé ces méthodes pour le calcul « a postériori » de louvrage, dans un esprit un peu différent. En effet nous avons voulu mettre à profit la possibilité d'étudier facilement l'influence de la variation des paramètres du calcul, afin d'obtenir un bon « calage \# sur les résultats expérimentaux.

Ces paramétres définissant les caractéristiques de l'ouvrage et du sol sont:

\section{La rigidité du rideau donnée par le produit $E \times 1$}

E : module d'élasticité de l'acier $E=2,1 \quad 10^{8} \mathrm{kN} / \mathrm{m}^{2}$ I: moment d'inertie pour lequel nous pouvons faire 2 hypothèses :

- palplanches indépendantes (fonctionnement indiqué par les jauges) soit I $=2 \times 11030 \mathrm{~cm}^{4} \mathrm{E} \times \mathrm{I}=46300 \mathrm{kN} \times$ $\mathrm{m}^{2}$

- palplanches encastrées au niveau des serrures (hypothèse de calcul habituelle) soit $1=72000 \mathrm{~cm}^{4}$ $\mathrm{E} \times \mathrm{I}=151200 \mathrm{kN} \times \mathrm{m}^{2}$

\section{Diagrammes limites de poussée et butée}

Ces caractéristiques de cisaillement utilisées figurent dans le tableau II.

Les diagrammes limites ont été calculés avec les coefficients de poussées et butées donnés par les tables de Caquot pour une obliquité :

$$
\begin{aligned}
& \delta=0 \text { pour les poussées } \\
& \delta=\frac{2}{3} \varphi \text { pour les butées }
\end{aligned}
$$

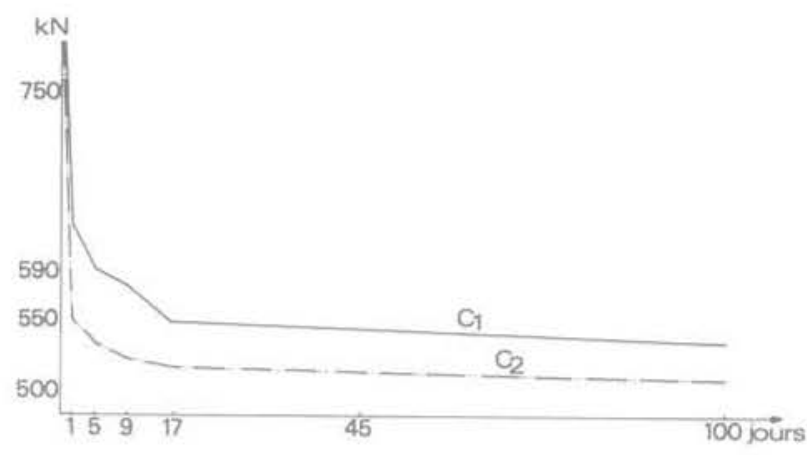

Fig. 16 Evolution de la tension des tirants précontraints

\section{La détermination de l'état initial}

On a fait l'hypothèse qu'avant battage, le terrain se trouve dans un état d'équilibre, le rideau ne doit alors être soumis à aucun effort juste après le battage.

Le diagramme de poussée côté berge est calculé en tenant compte de la présence du talus et on suppose qu'un diagramme symétrique l'équilibre côté Seine. On vérifie que ce diagramme avant est intermédiaire entre l'état de poussée minimale $K_{a}$ et de butée $K_{p}$.

Les madules de réaction

Ce paramètre joue un rôle important dans le calcul, mais c'est certainement celui dont la détermination est la plus difficile et la plus controversée.

\section{Nous avons utilisé deux approches:}

- Calcul des modules de réaction à partir des modules pressiométriques. La formule de calcul est celle définie par L. MENARD, mais ici nous avons calculé un module par couche de sol, en supposant à chaque fois que tout le milieu était constitué de cette couche (cette méthode, utilisée par de nombreux Bureaux d'Etudes, n'est pas du tout rigoureuse sur un plan théorique).

Cette méthode avait été appliquée pour le dimensionnement initial du rideau, les valeurs des modules étant par ailleurs définies plus arbitrairement pour les remblais d'apport (en l'absence d'essai, on avait tenu compte de la nature, du mode de compactage et de la largeur du " coin " en matériau remblayé).

- Détermination expérimentale des modules de réaction: Les modules de réaction ont également été déduits des mesures simultanées de pression et de déplacement. Mais cette méthode ne pouvait convenir que dans les zones où les déplacements étaient suffisamment élevés (>1 cm), c'est-à-dire dans les remblais et avec moins de précision dans les alluvions modernes.

Les modules obtenus selon ces deux méthodes sont donnés sur le tableau $n^{\circ}$ III.

\section{La prise en compte des tirants}

Les deux tirants équipés de cales ont été bloqués à des tensions de $750 \mathrm{kN}$. Toutefois après mise en tension des tirants adjacents, les cales ont indiqué des valeurs moyennes de tension de $510 \mathrm{kN}$.

$\mathrm{Ce}$ sont ces valeurs (effort horizontal de $221 \mathrm{kN} / \mathrm{ml}$, correspondant à un état d'équilibre, et compatibles avec le calcul bidimensionnel qui ont été prises en compte.

La rigidité du tirant, (tirant $10 \mathrm{~T} 13$ inclinés à $30^{\circ}$, espacés de $2 \mathrm{~m}$, de longueur libre $12 \mathrm{~m}$ ), pour les phases de calcul où il est considéré comme appui élastique, vaut : $\mathrm{K}=6930 \mathrm{kN} / \mathrm{ml} / \mathrm{m}$ 


\begin{tabular}{|c|c|c|c|c|c|}
\hline Nature & $\begin{array}{l}\text { Cotes NGF } \\
\mathrm{m}\end{array}$ & $\mathrm{kN} / \mathrm{m}^{3}$ & $\stackrel{\gamma^{\prime}}{\mathrm{kN} / \mathrm{m}^{3}}$ & $\underset{\mathrm{kPa}}{\mathrm{C}^{\prime}}$ & $\varphi^{\prime}$ \\
\hline $\begin{array}{l}\text { Remblais d'apport } \\
\text { (tout venant) }\end{array}$ & $\begin{array}{l}+29 \mathrm{a} \\
+21.20\end{array}$ & 17 & 9,5 & 0 & 35 \\
\hline $\begin{array}{l}\text { Alluvions modernes } \\
\text { sablo-vaseuses }\end{array}$ & $\begin{array}{l}+21,20 \mathrm{à} \\
+19,20\end{array}$ & 17 & 9,5 & 0 & 30 \\
\hline $\begin{array}{l}\text { Alluvions modernes } \\
\text { sablo-graveleuses }\end{array}$ & $\begin{array}{l}+19,20 \mathrm{a} \\
+15,50\end{array}$ & 17 & 9,5 & 0 & 37 \\
\hline $\begin{array}{l}\text { Alluvions anciennes } \\
\text { Sable et Graviers }\end{array}$ & $\begin{array}{l}+15,50 \mathrm{a} \\
+14,00\end{array}$ & 18 & 10 & 0 & 37 \\
\hline Sables de Beauchamp & $\begin{array}{l}+14,00 \mathrm{a} \\
+10\end{array}$ & 21 & 11 & 10 & 35 \\
\hline
\end{tabular}

Tableau 2 Caractéristiques utilisées pour les calculs des poussées et butées

\begin{tabular}{|c|c|c|c|c|}
\hline & \multirow[b]{2}{*}{ Cotes NGF } & \multicolumn{2}{|c|}{$\begin{array}{l}\text { Détermination du calcul } \\
\text { initial }\end{array}$} & \multirow{2}{*}{$\begin{array}{l}\text { Détermination } \\
\text { expérimentale } \\
\qquad \begin{array}{c}\mathrm{k}=\frac{\mathrm{p}}{\mathrm{y}} \\
\left(\mathrm{kN} / \mathrm{m}^{3}\right)\end{array}\end{array}$} \\
\hline & & $\begin{array}{l}\text { module } \\
\text { pressiome- } \\
\text { trique } \\
\text { (MPa) }\end{array}$ & $\begin{array}{c}\mathrm{K} \text { module de } \\
\text { réaction } \\
\left(\mathrm{kN} / \mathrm{m}^{3}\right)\end{array}$ & \\
\hline $\begin{array}{l}\text { Remblais hors } \\
\text { nappe }\end{array}$ & $\begin{array}{l}+29 a ̀+26 \\
+26 a+23,50\end{array}$ & $?$ & $\begin{array}{l}7000 \\
5000\end{array}$ & $\begin{array}{l}\text { entre } \\
1000\end{array}$ \\
\hline Remblais noyés & $+23,50 \mathrm{a}+21,20$ & $?$ & 3500 & et 2000 \\
\hline $\begin{array}{l}\text { Alluvions modernes } \\
\text { (sables vaseux) }\end{array}$ & $+21,20 a+19,20$ & 2 & 1000 & $\begin{array}{l}\text { entre } 1000 \\
\text { et } 1500\end{array}$ \\
\hline $\begin{array}{l}\text { Alluvions modernes } \\
\text { (sables graveleux) }\end{array}$ & $+19,20 \mathrm{a}+15,50$ & 6 & 7500 & \\
\hline Alluvions anciennes & $+15,50 \mathrm{à}+14,00$ & 10 & 15000 & \\
\hline $\begin{array}{l}\text { Sables de } \\
\text { Beauchamp }\end{array}$ & $+14,00 \mathrm{~d}+10,00$ & 15 & 15000 & \\
\hline
\end{tabular}

Tableau 3 Détermination des modules de réaction

\subsection{Ajustement des paramètres du calcul}

Avant de réaliser un dimensionnement complet de l'ouvrage par phases successives de travaux avec le programme RIDEAU*, nous avons réalisé une série de calcul dits de "dégrossissage " à l'aide d'un programme plus simple**, afin de tester l'influence des principaux paramètres.

- Le Programme RIDEAU a été mis à notre disposition par le B.E.T. DODIN, que nous tenons à remercier ici.

*.ll sagit du Programme SCPOU mis au point par le Bureau d'Etude de la Direction Départementale de l'Equipement de Seine Saint-Denis. Ce programme álastoplastique ne permet pas d'enchainer des phases successives de travaux.
Mais avant tout calcul, l'examen des résultats expérimentaux permet une simplification des données. En effet, les déformées successives du rideau montrent que :

- Dès la $1^{r 8}$ mise en tension du tirant du tirant et jusqu'au dragage les déplacements de la partie hors fiche du rideau se font vers le massif.

- Les déplacements de la partie fichée n'excèdent pas quelques millimètres, valeurs très insuffisantes pour mobiliser la butée limite du sol.

Ainsi, sur presque toute sa longueur, le sol se trouve dans un état d'équilibre élastique. Le module de réaction sera un paramètre essentiel du calcul, alors que les diagrammes limites de poussée et de butée n'interviendront pratiquement pas. 
Ne retenons que les principaux renseignements apportés par cette étude paramétrique :

1 - Les variations du module de réaction, influent directement sur les déformées (en première approximation, le déplacement en tête est inversement proportionnel à k). mais ont également une incidence importante sur les valeurs des moments fléchissants (cf tableau $n^{\circ}$ IV donnant deux exemples de calculs).

2 - L'augmentation de l'effort d'ancrage a pour effet d'augmenter le moment fléchissant en travée (cf tableau $\left.n^{\circ} \mathrm{V}\right)$.

Cela tient au fait, déjà constaté expérimentalement (cf, § 5-3) que le surcroît des réactions du sol est alors mobilisé essentiellement au dessous des tirants.

3 - Les valeurs des modules de réaction déduites des essais pressiométriques dans les différentes couches des terrains en place sont des ordres de grandeur raisonnables.

4 - Le module de réaction des remblais d'apports (matériau graveleux compacté, d'angle de frottement interne supérieur à 35 degrés) est bien inférieur aux estimations effectuées. Il est du même ordre de grandeur que celui des alluvions les plus médiocres $\mathrm{K}=1000$ à $2000 \mathrm{kN} / \mathrm{m}^{3}$.

5 - Le calcul ne fournit des valeurs de déformées, de pressions et de moments compatibles avec les données expérimentales que si l'on prend en compte l'inertie du rideau monolithique.

Ce résultat est assez déroutant car il est en contradiction avec la constatation de glissements dans les serrures mis en évidence par les jauges.

7.3 Calcul complet du rideau, tenant compte des phases de travaux (Programme RIDEAU)

Phases de travaux - caractéristiques de calcul

Le calcul considère les phases de travaux suivantes

Phase $n^{\circ} 1$ Remblaiement à l'arrière du rideau à +24 m NGF

Phase $n^{\circ} 2$ Première mise en tension à $150 \mathrm{kN}$ (effort appliqué $T=69 \mathrm{kN} / \mathrm{ml}$ )

Phase $n^{\circ} 3$ Remblaiement à $+26,50 \mathrm{~m}$ NGF (Le tirant est considéré comme appui élastique de rigidité $\mathrm{K}=6930 \mathrm{kN} / \mathrm{ml}$, avec tension initiale de $69 \mathrm{kN} / \mathrm{ml}$ ).

Phase $n^{\circ} 4 \quad$ Mise en tension définitive, application d'un effort de $221 \mathrm{kN} / \mathrm{ml}$.

Phases $n^{\circ} 4$ bis Simulation du cycle de tension et $n^{\circ} 4$ ter L'effort d'ancrage est porté à $314 \mathrm{kN} / \mathrm{ml}$ (phase 4 bis puis ramené à $221 \mathrm{kN} / \mathrm{ml}$ (phase 4 ter)).

Phase $n^{\circ} 5$ Remblaiement à $+29 \mathrm{~m} \mathrm{NGF}$ et dragage en Seine de $+21,20 \mathrm{~m} \dot{a}+19,20 \mathrm{~m}$ NGF.

Phase $n^{\circ} 6$ Application d'une surcharge en tête $10 \mathrm{kPa}$ et dénivellée de nappe de $1 \mathrm{~m}$ (cas de la décrue).

\section{Caractéristiques de calcul}

La rigidité est celle du rideau monolithique

$E \times I=151200 \mathrm{kN} \times \mathrm{m}^{2}$

Les modules de réaction valent :

$K=1500 \mathrm{kN} / \mathrm{m}^{3}$ dans le Remblai

$\mathrm{K}=1000 \mathrm{kN} / \mathrm{m}^{3}$ dans les Alluvions sablo-vaseuses

$(+21,20$ à $+19,20)$

\begin{tabular}{|c|c|c|}
\hline & $1^{\text {er }}$ Cas & $2^{\circ}$ Cas \\
\hline \multicolumn{3}{|c|}{ - Module de réaction en $\mathrm{kN} / \mathrm{m}^{3}$} \\
\hline $\begin{array}{l}+26,50 \text { à } 21,20(\mathrm{R}) \\
+21,20 \text { à } 15,50 \\
+15,50 \text { à }+14(\mathrm{Aa}) \\
+14 \text { à } 10(\mathrm{SB})\end{array}$ & $\begin{array}{r}2000 \\
1000 \\
5000 \\
10000\end{array}$ & $\begin{array}{r}7500 \\
5000 \\
15000 \\
15000\end{array}$ \\
\hline - Déformée en tête & $6 \mathrm{~cm}$ & $2,5 \mathrm{~cm}$ \\
\hline $\begin{array}{l}\text { - Déformée au niveau } \\
\text { du tirant }\end{array}$ & $3,6 \mathrm{~cm}$ & $1.4 \mathrm{~cm}$ \\
\hline $\begin{array}{l}\text { - Moment fléchissant } \\
\text { maximum }\end{array}$ & $\begin{array}{c}273 \mathrm{mkN} \\
\mathrm{a} \\
+20 \mathrm{~m} \mathrm{NGF}\end{array}$ & $\begin{array}{c}150 \mathrm{mkN} \\
\text { à } \\
21,20 \mathrm{~m} \mathrm{NGF}\end{array}$ \\
\hline
\end{tabular}

Tableau 4 Influence des variations des modules de réaction sur les déformées et les moments fléchissants Caractéristiques communes aux calculs :

Remblaiement aे + 26,50 m NGF

Effort d'ancrage $324 \mathrm{kN} / \mathrm{ml}$ (750 kN par tirant)

$E \times I=151200 \mathrm{kN} \cdot \mathrm{m}^{2}$

\begin{tabular}{l|c|c}
\hline & $\begin{array}{c}1^{\text {er }} \mathrm{Cas} \\
\mathrm{T}=221 \mathrm{kN} / \mathrm{ml}\end{array}$ & $\begin{array}{c}2^{\circ} \mathrm{Cas} \\
\mathrm{T}=324 \mathrm{kN} / \mathrm{ml}\end{array}$ \\
\hline $\begin{array}{l}\text { Effort dans le tirant } \\
\text { Déformée en tête }\end{array}$ & $310 \mathrm{kN}$ & $750 \mathrm{kN}$ \\
$\begin{array}{l}\text { Déformée au niveau } \\
\text { du tirant }\end{array}$ & $2 \mathrm{~cm}$ & $3.6 \mathrm{~cm}$ \\
$\begin{array}{l}\text { Moment fléchissant maxi } \\
\begin{array}{l}\text { Moment fléchissant au } \\
\text { niveau du tirant }\end{array}\end{array}$ & $189 \mathrm{mkN}$ & $270 \mathrm{mkN}$ \\
\hline
\end{tabular}

Tableau 5 Influence de la valeur de la tension d'ancrage (données communes: Remblaiement à + 26,5 m NGF, $E \times I=151200 \mathrm{kN} \cdot \mathrm{m}^{2}$ ) Modules de réaction:

$K=2000 \mathrm{kN} / \mathrm{m}^{3}$ dans $R$ et $\mathrm{Am}$

$K=10000 \mathrm{kN} / \mathrm{m}^{3}$ dans $A a$

$K=100000 \mathrm{kN} / \mathrm{m}^{3}$ dans $S B$

$\mathrm{K}=5000 \mathrm{kN} / \mathrm{m}^{3}$ dans les Alluvions sablo-graveleuses $(+19,20 \mathrm{a}+15,50)$

$\mathrm{K}=10000 \mathrm{kN} / \mathrm{m}^{3}$ dans les Alluvions anciennes

$K=50000 \mathrm{kN} / \mathrm{m}^{3}$ dans les Sables de Beauchamp. 


\begin{tabular}{|c|c|c|c|}
\hline & Phase $n^{\circ} 4$ & Phase $n^{\circ} 4$ bis & Phase $n^{\circ} 4$ ter \\
\hline Effort dans le tirant & $221 \mathrm{kN} / \mathrm{ml}$ & $324 \mathrm{kN} / \mathrm{ml}$ & $221 \mathrm{kN} / \mathrm{ml}$ \\
\hline Déplacement en tête & $4,3 \mathrm{~cm}$ & $6,7 \mathrm{~cm}$ & $5.1 \mathrm{~cm}(+17 \%)$ \\
\hline $\begin{array}{l}\text { Déplacement au niveau } \\
\text { du tirant }\end{array}$ & $2,7 \mathrm{~cm}$ & $4,2 \mathrm{~cm}$ & $3,1 \mathrm{~cm}$ \\
\hline $\begin{array}{l}\text { Moment fléchissant } \\
\text { au niveau du tirant }\end{array}$ & $-55,5 \mathrm{kN} / \mathrm{ml}$ & $-74 k N$ & $-33 \mathrm{kN}$ \\
\hline $\begin{array}{l}\text { Moment fléchissant } \\
\text { maximal }\end{array}$ & $\begin{array}{c}191 \mathrm{mkN} / \mathrm{ml} \\
\mathrm{a}+21,20 \mathrm{~m} \mathrm{NGF}\end{array}$ & $265 \mathrm{mkN} / \mathrm{ml}$ & $\begin{array}{c}220 \mathrm{mkN} / \mathrm{ml} \\
(+15 \%)\end{array}$ \\
\hline
\end{tabular}

Tableau 6 Simulation d'un cycle de mise en tension. Evolution des déplacements et des moments fléchissants

Prise en compte de l'hystérésis du sol

Simulation du cycle de mise en tension

Le programme utilisé tient compte d'une hystérésis du sol, c'est-à-dire la non réversibilité des déplacements dans le domaine plastique.

La simulation approximative par le calcul d'un cycle de mise en tension (phases $4-4$ bis et 4 ter) permet d'analyser le comportement du sol suivant un cycle d'hystérésis. (tableau VI et fig. $n^{\circ}$ 17)

On constate que les déformées ainsi que le moment fléchissant maximal se trouvent majorés d'environ $15 \%$ à l'issue de cycle de tension.

L'augmentation de la tension de $221 \mathrm{kN}$ (phase 4) à 324 $\mathrm{kN}$ (Phase 4 bis) provoque une plastification des sols en partie supérieure. Lorsque l'on revient à l'état initial, (phase 4 ter) la pression exercée par le sol est plus faible pour une déformée accrue (fig. $n^{\circ} 17$ a) le cycle de tension a entrainé une sorte de "détente " du sol en partie supérieure.

Sous le tirant par contre, le sol reste en équilibre élastique, en fin de cycle (fig. 17b), les déformées et pressions ont augmenté.

Le centre de gravité des réactions du sol s'est déplacé vers le bas ce qui a pour effet d'augmenter le moment fléchissant en travée.

Comparaison des résultats du calcul global et du calcul par phase

Nous avons comparé les résultats du calcul par phases successives $1,2,3$ et 4 avec les résultats d'un calcul global (fig. 18 tableau VII).

Les diagrammes des pressions diffèrent sensiblement entre les deux calculs.

Comme dans la simulation du cycle de mise en tension, on constate que la prise en compte de phases intermédiaires des travaux (avec alternance des directions d'efforts) se traduit par une majoration relativement importante des déformée $(+30 \%)$ et des moments fléchissants $(+22 \%)$.
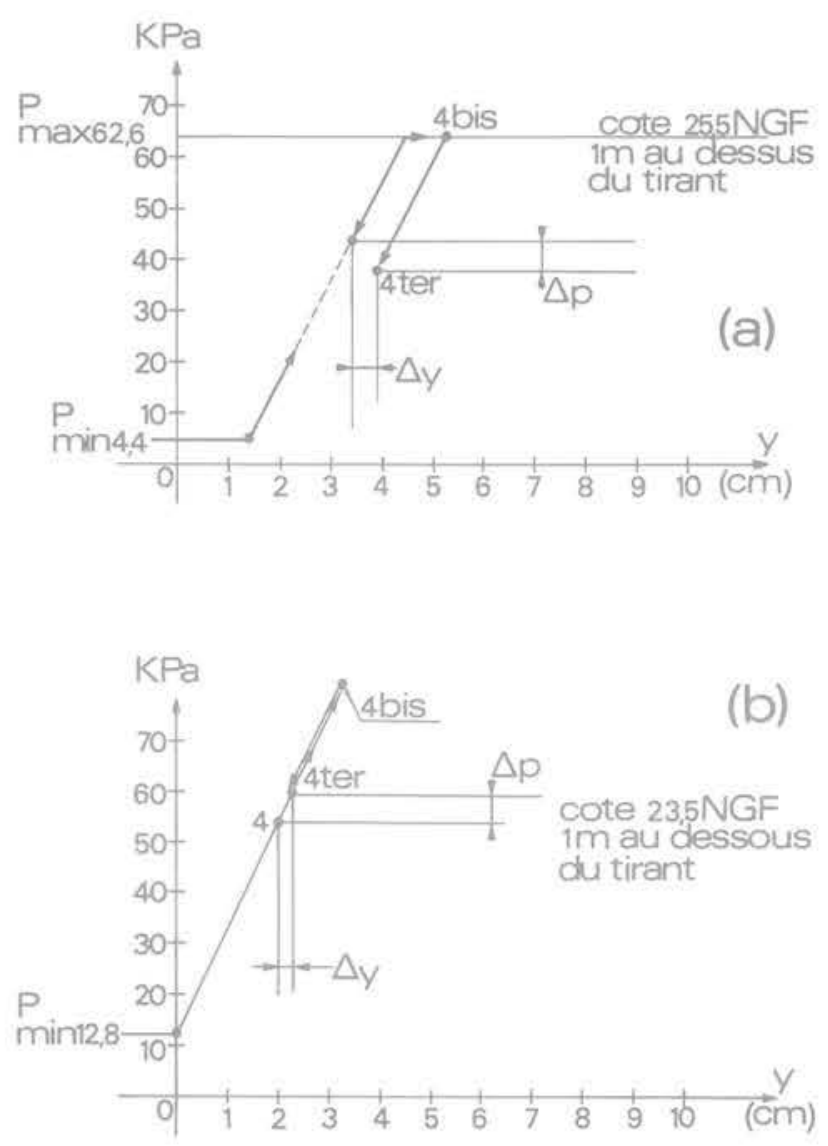

Fig. 17 Schéma expliquant l'évolution des pressions sur le cycle d'hystérésis 


\section{DEFORMEES}

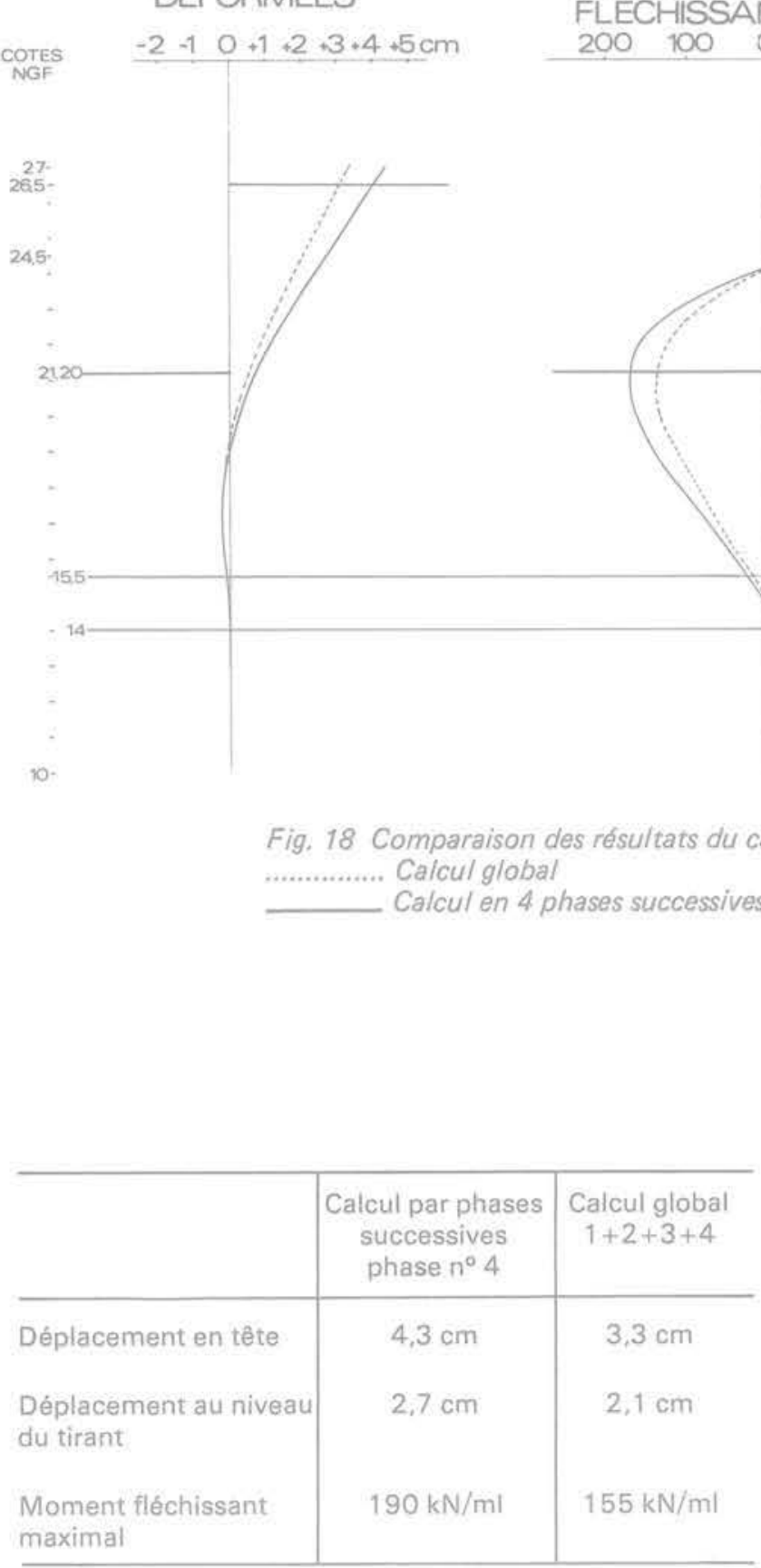

Tableau 7
MOMENTS FLECHISSANTS

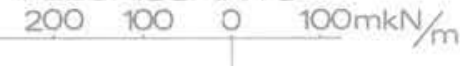

\section{PRESSIONS}

$\begin{array}{llllll}40 & 20 & 0 & 20,40 \quad 60 \quad \mathrm{kPa}\end{array}$




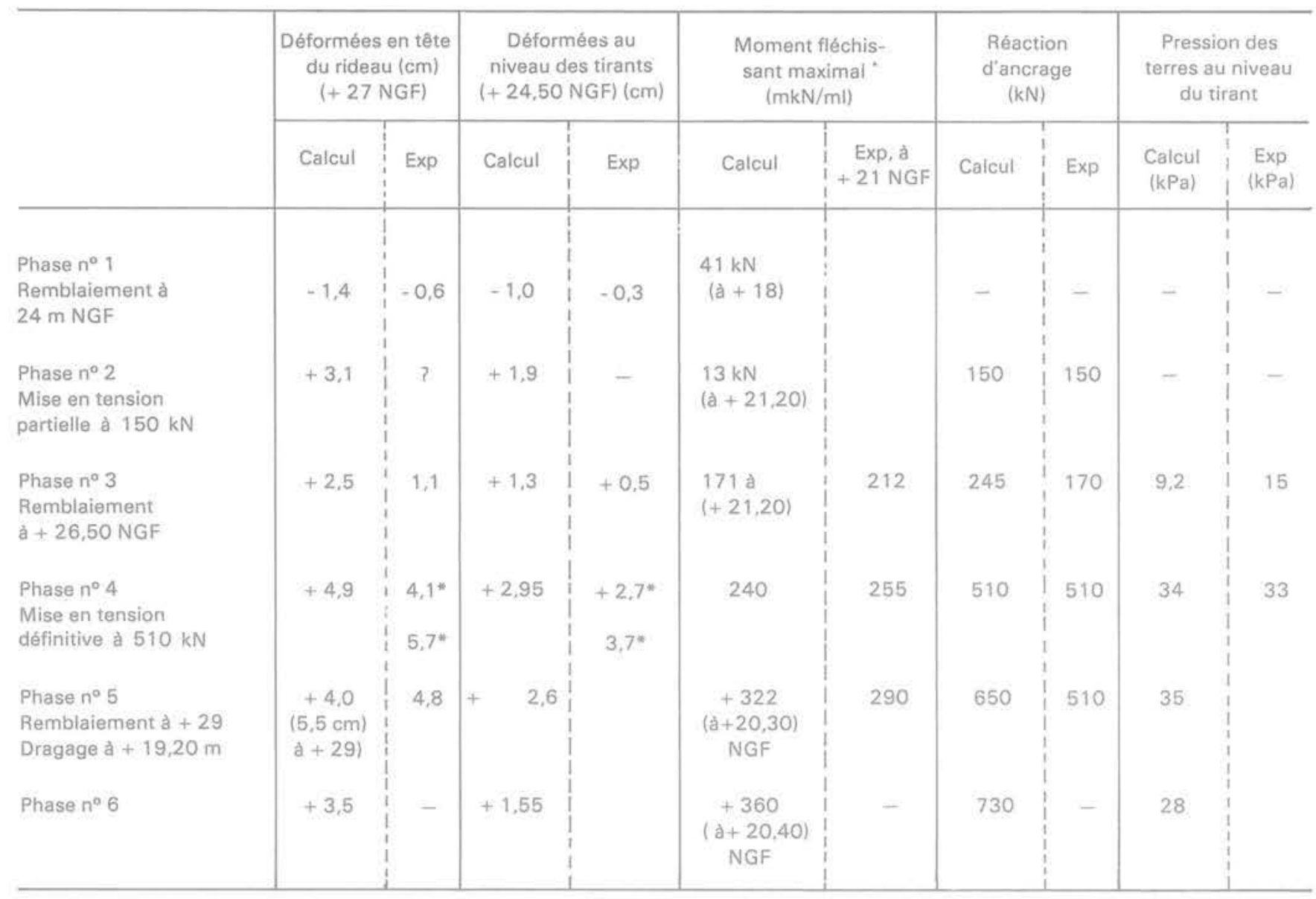

- 1re valeur - au moment de la mise en tension

$2^{\circ}$ valeur - six semaines plus tard

\section{Tableau 8}

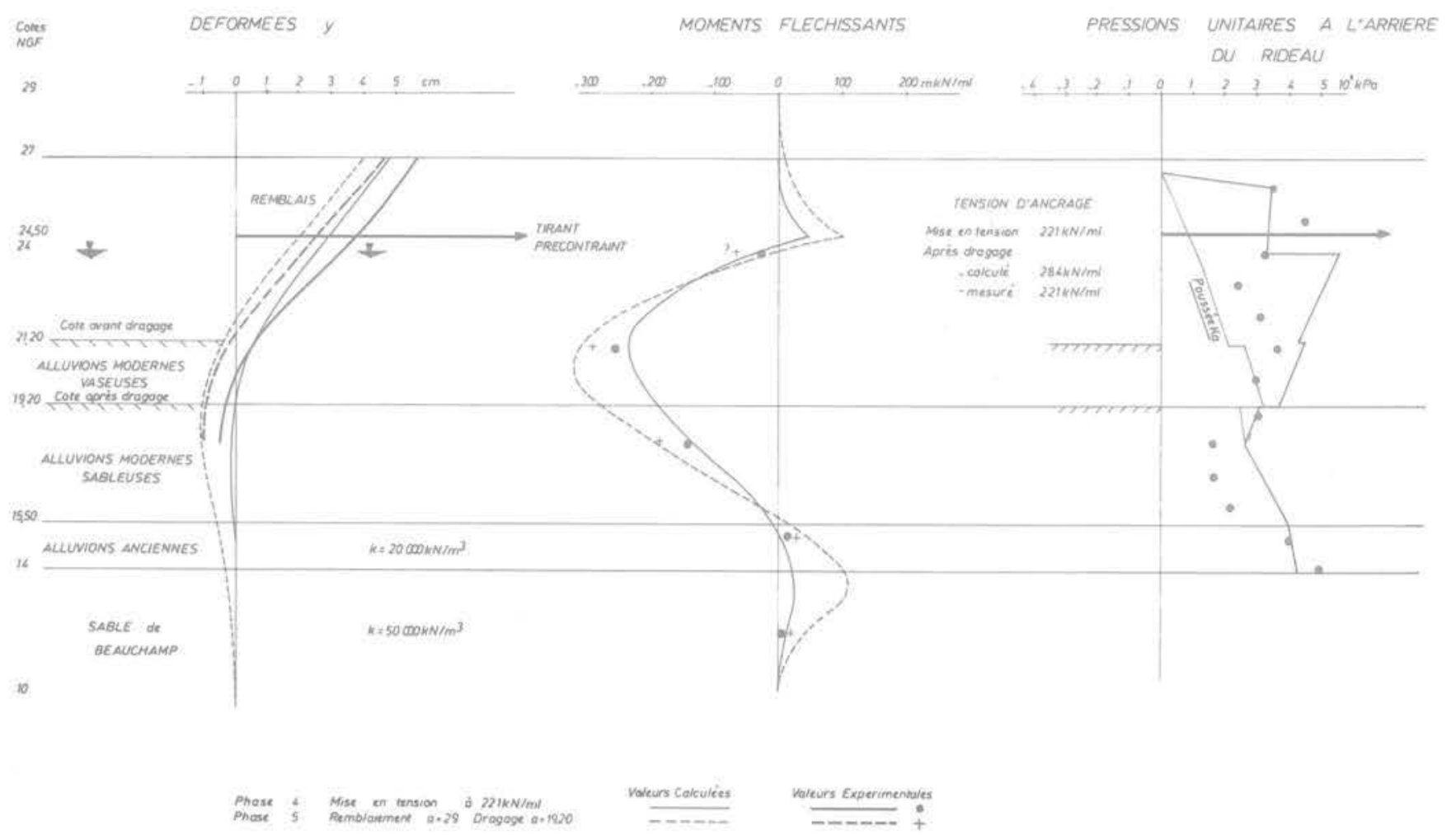

Fig. 19 Comparaison des résultats expérimentaux et des valeurs calculées pour 2 phases de travaux 


\section{Conclusion}

Grâce aux enseignements de quelques tentatives antérieures, cette expérimentation d'un rideau de palplanches a pu se dérouler de manière satisfaisante et a permis de mesurer tout au long de la construction à la fois les déformées de l'ouvrage, les contraintes dans l'acier et les pressions des terres.

Le premier intérêt de cette étude en vraie grandeur est de mettre en évidence le fonctionnement réel des palplanches, qui s'avère assez différent des schémas de calcul, assimilant le rideau à un écran plan continu :

- ainsi, les pressions des terres mesurées dans le creux des palplanches (dans des remblais rapportés) sont beaucoup plus faibles que celles trouvées en bosse.

- par ailleurs, les mesures de contraintes obtenues à partir de jauges situées à la fois sur les âmes et près des serrures révèlent un fonctionnement quasi indépendant des deux palplanches d'une paire, par suite d'un glissement relatif au niveau de la serrure. Concrètement, les observations faites militent en faveur d'une liaison des palplanches entre elles par pinçage ou soudage.

Naturellement, ces éléments viennent sérieusement compliquer l'interprétation des mesures, tant pour la vérification des équations d'équilibre du rideau que pour la détermination des moments fléchissants.

Le calcul "élastoplastique " à postériori de l'ouvrage mené sous forme d'une étude paramétrique, avait avant tout pour but d'appréhender les valeurs du module de réaction horizontal du sol, paramètre mal connu. Il est apparu qu'ici les règles de calcul indiquées par L. MENARD en fournissent un ordre de grandeur satisfaisant, ce résultat ne pouvant cependant pas être généralisé compte tenu de l'hétérogénéité du site.
Une opinion fort répandue est qu'une forte erreur d'appréciation du module de réaction a une incidence importante sur les prévisions des déformées, mais faible sur celle des efforts dans le rideau. Le calcul a confirmé les résultats expérimentaux montrant que ce raisonnement n'était pas généralisable au dimensionnement des rideaux ancrés par tirants actifs et faisant appel à un module de réaction dans la partie hors fiche. En effet la répartition des réactions de sols mobilisées lors des mises en tension dépend largement de ce module et peut avoir une répercussion importante sur les moments fléchissants.

En définitive, si cette expérimentation a apporté d'utiles renseignements sur le fonctionnement d'un rideau ancré, elle a peut-être soulevé davantage de problèmes qu'elle n'en a résolu, c'est bien la preuve que dans ce domaine du dimensionnement des rideaux de soutènement, nous avons encore beaucoup à apprendre.

\section{Références bibliographiques}

JOSSEAUME - GIGAN - FERRAND - HOUY (1977) Mesures et observations sur rideaux de palplanches - Colloque Maitre d'ouvrages - Producteurs de palplanches métalliques Paris avril 1977

Dr LOHMEYER (1937) - A propos du module de résistance des palplanches métalliques en U-Bautechnik - décembre 1937.

GIGAN J.P. - Expérimentation d'un rideau de palplanches Voie rive gauche de Seine à Asnières - LPC - Rapport de Recherche à paraitre. 